\title{
RELAXATION METHODS FOR NETWORK FLOW PROBLEMS WITH CONVEX ARC COSTS*
}

\author{
DIMITRI P. BERTSEKAS $\dagger$, PATRICK A. HOSEIN $\dagger$ AND PAUL TSENG $\dagger$
}

\begin{abstract}
We consider the standard single commodity network flow problem with both linear and strictly convex possibly nondifferentiable are costs. For the case where all are costs are strictly convex we study the convergence of a dual Gauss-Seidel type relaxation method that is well suited for parallel computation. We then extend this method to the case where some of the arc costs are linear. As a special case we recover a relaxation method for the linear minimum cost network flow problem proposed in Bertsekas [1] and Bertsekas and Tseng [2].
\end{abstract}

Key words. network flow, relaxation methods, parallel computation

1. Introduction. Consider a directed graph with set of nodes $N$ and set of arcs $A$. We will write $j \sim(i, k)$ to denote that the start and end nodes of arc $j$ are $i$ and $k$, respectively. The network incidence matrix is denoted by $E$ and has elements $e_{i j}$ given by

$$
e_{i j}=\left[\begin{array}{ll}
1 & \text { if } i \text { is the start node of } \operatorname{arc} j, \\
-1 & \text { if } i \text { is the end node of } \operatorname{arc} j \\
0 & \text { otherwise. }
\end{array}\right.
$$

We denote by $x_{j}$ the flow of arc $j$, and by $d_{i}$ the deficit of node $i$ which is defined by

$$
d_{i}=\sum_{j \in A} e_{i j} x_{j} \quad \forall i \in N
$$

In words $d_{i}$ is the balance of flow outgoing from $i$ and flow coming into $i$. The vectors with coordinates $x_{j}$ and $d_{i}$ are denoted $x$ and $d$ respectively. Thus (2) is written as

$$
d=E x \text {. }
$$

In what follows the association of particular deficit vectors and flow vectors via (3) should be clear from the context.

Each arc $j$ has associated with it a cost function $f_{j}: R \rightarrow(-\infty,+\infty]$. We consider the problem of minimizing total cost subject to a conservation of flow constraint at each node:

$$
\begin{aligned}
& \text { minimize } f(x)=\sum_{j \in A} f_{j}\left(x_{j}\right) \\
& \text { subject to } x \in C
\end{aligned}
$$

where $C$ is the circulation subspace:

$$
C=\left\{x \mid d_{i}=0, i \in N\right\}=\{x \mid E x=0\} .
$$

We make the following assumptions on $f_{j}$.

Assumption A. Each function $f_{j}$ is convex, lower semicontinuous, and there exists at least one feasible solution for problem (4), i.e., the effective domain of $f$

$$
\operatorname{dom}(f)=\{x \mid f(x)<+\infty\}
$$

and the circulation subspace $C$ have a nonempty intersection.

* Received by the editors January 6,1986; accepted for publication (in revised form) September 3 , 1986. This work was supported by the National Science Foundation under grant NSF-ECS-8217668.

$\dagger$ Laboratory for Information and Decision Systems, Massachusetts Institute of Technology, Cambridge, Massachusetts 02139. 
Assumption B. The conjugate convex function of each $f_{j}$ defined by

$$
g_{j}\left(t_{j}\right)=\sup _{x_{j}}\left\{t_{j} x_{j}-f_{j}\left(x_{j}\right)\right\}
$$

is real valued, i.e., $-\infty<g_{j}\left(t_{j}\right)<+\infty$ for all $t_{j} \in R$.

Assumption B implies that $f_{j}\left(x_{j}\right)>-\infty$ for all $x_{j}$ and $j$. It follows that the set of points where $f_{j}$ is real valued, denoted dom $\left(f_{j}\right)$, is a nonempty interval the right and left endpoints of which (possibly $+\infty$ or $-\infty$ ) we denote by $c_{j}$ and $l_{j}$, respectively, i.e.,

$$
c_{j}=\sup \left\{x_{j} \mid f_{j}\left(x_{j}\right)<+\infty\right\}, \quad l_{j}=\inf \left\{x_{j} \mid f_{j}\left(x_{j}\right)<+\infty\right\} .
$$

We call $c_{j}$ and $l_{j}$ the upper and lower capacity bounds of $f_{j}$ respectively. It is easily seen that Assumptions A and B imply that for every $t_{j}$ there is some $x_{j} \in \operatorname{dom}\left(f_{j}\right)$ attaining the supremum in (6), and furthermore

$$
\lim _{\left|x_{j}\right| \rightarrow+\infty} f_{j}\left(x_{j}\right)=+\infty
$$

It follows that the cost function of (4) has bounded level sets, and therefore (using also the lower semicontinuity of $f$ ) there exists at least one optimal flow vector.

Assumptions $\mathrm{A}$ and $\mathrm{B}$ are satisfied, if, for example, $f_{j}$ is of the form

$$
f_{j}\left(x_{j}\right)= \begin{cases}\hat{f}_{j}\left(x_{j} .\right. & \text { if } x_{j} \in\left[l_{j}, c_{j}\right] \\ \infty & \text { otherwise }\end{cases}
$$

where $l_{j}, c_{j}$ are given upper and lower bounds on the flow of arc $j$, and $\hat{f}_{j}$ is a real valued convex function on the real line $R$. In this case $g_{j}\left(t_{j}\right)$ is linear for $\left|t_{j}\right|$ large enough with slopes $l_{j}$ and $c_{j}$ as $t_{j}$ approaches $-\infty$ and $+\infty$, respectively (see Fig. 1.1).

Problem (4) is called the optimal distribution problem in Rockafellar [3]. The same reference develops in detail a duality theory (a refinement of what can be obtained from Fenchel's duality theorem) involving the dual problem

$$
\begin{array}{ll}
\text { minimize } & g(t) \stackrel{\Delta}{=} \sum_{j \in A} g_{j}\left(t_{j}\right) \\
\text { subject to } & t \in C^{\perp}
\end{array}
$$

where $t$ is the vector with coordinates $t_{j}, j \in A$, and $C^{\perp}$ is the orthogonal complement of $C$. We call $t_{j}$ the tension of the arc $j$ and $C^{\perp}$ the tension subspace. From (1)-(3) and

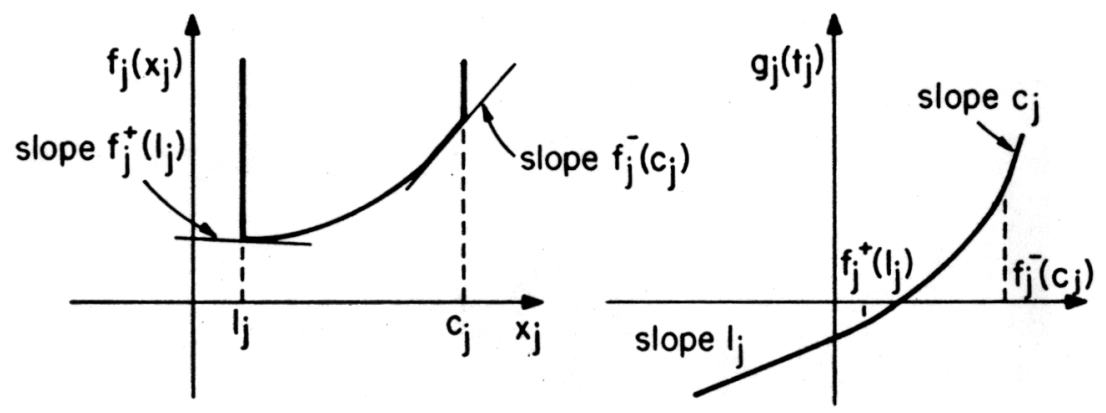

FIG. 1.1. Primal cost function of the form (7) and its dual. 
(5) we have that $t \in C^{\perp}$ if and only if there exist scalars $p_{i}, i \in N$, called prices, such that

$$
t_{j}=p_{i}-p_{k} \quad \forall j \in A \text { with } j \sim(i, k),
$$

or equivalently

$$
t=E^{T} p
$$

where $E^{T}$ is the transpose of the network incidence matrix $E$ and $p$ is the vector with coordinates $p_{i}, i \in N$. Therefore the dual problem (8) can also be written as

$$
\begin{aligned}
& \text { minimize } q(p) \\
& \text { subject to no constraints on } p
\end{aligned}
$$

where $q$ is the dual functional

$$
q(p)=\sum_{\substack{j \in A \\ j \sim(i, k)}} g_{j}\left(p_{i}-p_{k}\right)
$$

As shown in [3, p. 349], Assumption A guarantees that there is no duality gap in the sense that the primal and dual optimal costs are opposites of each other.

An important fact for the purposes of the present paper is that (in view of Assumption B above) the dual problem (11) is an unconstrained optimization problem. If each function $f_{j}$ is strictly convex, the dual functional is also differentiable ([4, p. 253]) and as a result unconstrained smooth optimization methods can be applied for solution. This is particularly so since the gradient of the dual cost can be easily calculated. Indeed, when $f_{j}$ is strictly convex, for every tension vector $t$ there exists a unique flow vector $x$ such that

$$
x_{j}=\arg \max _{z_{j}}\left\{t_{j} z_{j}-f_{j}\left(z_{j}\right)\right\} \quad \forall j \in A
$$

and it can be shown $\left[4\right.$, p. 218] that $x_{j}$ is the gradient of $g_{j}$ at $t_{j}$

$$
x_{j}=\nabla g_{j}\left(t_{j}\right) \quad \forall j \in A .
$$

From (1) and (12) we see that for a given price vector $p$ the partial derivatives of the dual functional $q$ are given by

$$
\frac{\partial q(p)}{\partial p_{i}}=\sum_{j \in A} e_{i j} \nabla g_{j}\left(t_{j} \quad \forall i \in N\right.
$$

Equivalently (cf. (2)), the partial derivative $\partial q(p) / \partial p_{i}$ equals the deficit of node $i$ when the arc flows $x_{j}$ are the unique scalars defined by (13).

The differentiability of the dual cost when the primal cost is strictly convex motivates a Gauss-Seidel type of algorithm whereby, given a price vector $p$, one calculates the corresponding flows $x_{j}=\nabla g_{j}\left(t_{j}\right), j \in A$, chooses a node $i$ with positive (negative) deficit and decreases (increases) $p_{i}$ up to the point where the corresponding partial derivative $\partial q / \partial p_{i}$ becomes zero. (This amounts to minimizing the dual functional $q$ along the coordinate $p_{i}$.) One then repeats the procedure iteratively. The algorithm above is attractive not only because of its simplicity but also because it lends itself naturally to distributed computation, whereby minimization along different price coordinates is carried out simultaneously by several processors. Indeed, this can be done 
in an asynchronous format as described and analyzed in Bertsekas and El Baz [5]. Simulations of a synchronous parallel method of this type [19] have shown remarkable speedup in computation time.

Gauss-Seidel relaxation methods for unconstrained optimization have been studied extensively [6]-[10]. However they typically require for convergence something like a strict convexity assumption on the cost minimized as well as boundedness of its level sets (see [10] for a counterexample). Unfortunately the dual cost (12) always has unbounded level sets since adding the same constant to all node prices leaves the cost unchanged. Even if we remove this degree of freedom by restricting the price of some special node to be zero (i.e. passing to a quotient space), the dual cost may still have unbounded levels sets and is not strictly convex when the functions $f_{j}$ are nondifferentiable as in the important special case (7) where they imply capacity constraints. One contribution of the present paper $(\S 2)$ is to show convergence of a flow sequence generated by the Gauss-Seidel method to the unique optimal solution of the primal problem (4). Convergence of the corresponding price vector sequence to some optimal solution of the dual problem (11) is also shown assuming the dual has an optimal solution. For this we actually require that the minimization along coordinates be done only approximately. Furthermore nodes can be relaxed in arbitrary order. The only requirement is that each node is relaxed infinitely often. This result is new and is remarkable in that it requires a rather unconventional method of proof. It improves on a result by Pang [11] (see also an earlier paper by Cottle and Pang [12]) which asserts convergence of the flow vector sequence under the assumption that $\boldsymbol{g}_{j}$ is of the form (7) with $\hat{f}_{j}$ differentiable, and strongly convex (rather than just strictly convex as we assume). Pang's result requires exact minimization along each coordinate and contains no assertion on convergence of the price vector sequence; however it applies to a more general problem where the primal cost function need not be separable and the linear constraints need not have a network structure. The paper by Cottle and Pang [12] asserts subsequence convergence to a dual optimal solution for a transportation problem with quadratic arc costs but also uses a nondegeneracy assumption and places a restriction in the way relaxation is carried out. This result is strengthened in our analysis as described above.

When some of the arc cost functions $f_{j}$ are not strictly convex, the dual cost is not differentiable, and the Gauss-Seidel method breaks down. However Bertsekas [1] and Bertsekas and Tseng [2] have proposed methods that are conceptually related to Gauss-Seidel and work with linear arc costs. They allow line minimization along directions involving several coordinates to cope with situations where minimizing along a single coordinate is not possible. Computational experimentation with standard benchmark problems and a code named RELAX [1], [2] shows that these methods are very promising and outperform, in terms of computation time, some of the best primal simplex and primal dual codes currently available. The second objective of this paper is to propose in $\S 3$ a new relaxation method that in some sense bridges the gap between the strictly convex arc cost Gauss-Seidel method described earlier and the Bertsekas-Tseng linear arc cost version. We show that this method works with both linear and nonlinear (convex) arc costs and contains as special cases both relaxation methods described above. To our knowledge the only other known algorithm for network problems with both linear and nonlinear, possibly nondifferentiable, arc costs is Rockafellar's fortified descent method [3, Chap. 9]. Our algorithm relates in roughly the same way to the Bertsekas-Tseng relaxation method, as Rockafellar's relates to the classical primal-dual method. We note that the methods considered here for linear costs and, more generally, not strictly convex costs are not easily parallelizable. Related 
synchronous and asynchronous relaxation methods that admit massive parallelization have been proposed recently in [20], [21].

The last section of the paper provides results of computational experimentation with codes implementing both of the relaxation algorithms proposed.

2. The relaxation method for strictly convex arc costs. In this section in addition to Assumptions $\mathrm{A}$ and $\mathrm{B}$, there will be a standing assumption that each $f_{j}$ is strictly convex. Two important consequences of this assumption are that the optimal flow vector is unique and the conjugate functions $g_{j}$ are differentiable (in addition to being real valued by Assumption B). Indeed it is easily verified (see also [3] and [4, p. 218]) that we have for all $t_{j}$

$$
\nabla g_{j}\left(t_{j}\right)=\arg \max _{x_{j}}\left\{t_{j} x_{j}-f_{j}\left(x_{j}\right)\right\} .
$$

Furthermore $\nabla g_{j}\left(t_{j}\right)$ is the unique scalar $x_{j}$ satisfying together with $t_{j}$ the Complementary Slackness $(\mathrm{CS})$ condition

$$
f_{j}^{-}\left(x_{j}\right) \leqq t_{j} \leqq f_{j}^{+}\left(x_{j}\right)
$$

where $f_{j}^{-}\left(x_{j}\right)$ and $f_{j}^{+}\left(x_{j}\right)$ denote the left and right derivatives of $f_{j}$ at $x_{j}$ (see Fig. 2.1). These derivatives are defined in the usual way for $x_{j}$ in the interior of $\operatorname{dom}\left(f_{j}\right)$. When $-\infty<l_{j}<c_{j}$ we define

$$
f_{j}^{+}\left(l_{j}\right)=\lim _{\xi \downarrow l_{j}}{ }_{i}^{+}(\xi), \quad f_{j}^{-}\left(l_{j}\right)=-\infty
$$

When $l_{j}<c_{j}<+\infty$ we define

$$
f_{j}^{-}\left(c_{j}\right)=\lim _{\xi \uparrow c_{j}} f_{j}^{-}(\xi), \quad f_{j}^{+}\left(c_{j}\right)=+\infty .
$$

Finally when $l_{j}=c_{j}$ we define $f_{j}^{-}\left(l_{j}\right)=-\infty, f_{j}^{+}\left(c_{j}\right)=+\infty$. Note that $\nabla g_{j}\left(t_{j}\right)$ is continuous and monotonically nondecreasing. We define the deficit functions $d_{i}$ by

$$
d_{i}(p)=\sum_{j \in A} e_{i j} \nabla g_{j}\left(t_{j}\right) \quad \forall i \in N
$$

where $t=E^{r} p$, and denote by $d(p)$ the vector with coordinates $d_{i}(p)$. Note that the definition of $d$ is identical to that given in (2), except that here we have used the strict convexity of $f_{j}$ to express flow and deficit as functions of the dual price vector. In view of the form of the dual functional, the relation above yields

$$
d_{i}(p)=\frac{\partial q(p)}{\partial p_{i}} \quad \forall i \in N .
$$

Since $d_{i}(p)$ is a partial derivative of a differentiable convex function, we have that $d_{i}(p)$ is continuous and monotonically nondecreasing in the coordinate $p_{i}$.

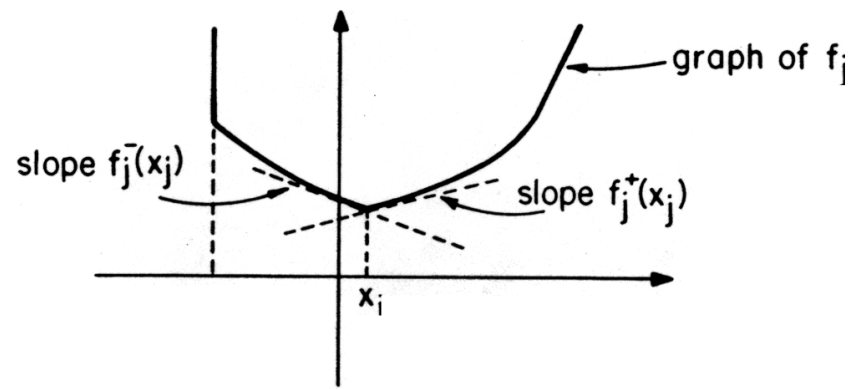

Fig. 2.1. The left and right derivatives of $f_{j}$. 

we have

Proof. Fix an index $r \geqq 0$. Denote $s=s^{r}$ and $\Delta=p_{s}^{r+1}-p_{s}^{r}$. From (6), (12) and (16)

$$
q\left(p^{r}\right)=\sum_{j \in A}\left[t_{j}^{r} x_{j}^{r}-f_{j}\left(x_{j}^{r}\right)\right] \quad \forall r \geqq 0 .
$$

Therefore

$$
\begin{aligned}
q\left(p^{r}\right)-q\left(p^{r+1}\right) & =\sum_{j \in A}\left[t_{j}^{r} x_{j}^{r}-f_{j}\left(x_{j}^{r}\right)\right]-\sum_{j \in A}\left[t_{j}^{r+1} x_{j}^{r+1}-f_{j}\left(x_{j}^{r+1}\right)\right] \\
& =\sum_{j \in A}\left[t_{j}^{r} x_{j}^{r}-f_{j}\left(x_{j}^{r}\right)\right]-\sum_{j \in A}\left[\left(t_{j}^{r}+e_{s j} \Delta\right) x_{j}^{r+1}-f_{j}\left(x_{j}^{r+1}\right)\right] \\
& =\sum_{j \in A}\left[f_{j}\left(x_{j}^{r+1}\right)-f_{j}\left(x_{j}^{r}\right)-\left(x_{j}^{r+1}-x_{j}^{r}\right) t_{j}^{r}-e_{s j} \Delta x_{j}^{r+1}\right] \\
& =\sum_{j \in A}\left[f_{j}\left(x_{j}^{r+1}\right)-f_{j}\left(x_{j}^{r}\right)-\left(x_{j}^{r+1}-x_{j}^{r}\right) t_{j}^{r}\right]-\Delta \sum_{j \in A} e_{s j} x_{j}^{r+1} \\
& =\sum_{j \in A}\left[f_{j}\left(x_{j}^{r+1}\right)-f_{j}\left(x_{j}^{r}\right)-\left(x_{j}^{r+1}-x_{j}^{r}\right) t_{j}^{r}\right]-\Delta d_{s}\left(p^{r+1}\right) .
\end{aligned}
$$

Since $\Delta d_{s}\left(p^{r+1}\right) \leqq 0$ (and $d_{s}\left(p^{r+1}\right)=0$ if we use line minimization) the left side of (18) follows. The right side of (18) follows from the strict convexity of $f_{j}$ and the fact $\boldsymbol{x}^{r+1} \neq \boldsymbol{x}^{r}$. QED

Proposition 2.2. The sequence $\left\{x^{r}\right\}$ is bounded.

Proof. We first note that at every iteration the total deficit does not increase, i.e.,

$$
\sum_{i \in N}\left|d_{i}\left(p^{r+1}\right)\right| \leqq \sum_{i \in N}\left|d_{i}\left(p^{r}\right)\right|
$$

(This follows from the fact that a flow change on an arc reflects itself in a change of the deficit of its start node and an opposite change in the deficit of its end node. Furthermore the deficit of node $s^{r}$ chosen for relaxation at the $r$ th iteration cannot increase in absolute value or change sign during that iteration.) It follows that $\left\{d\left(p^{r}\right)\right\}$ is bounded. We now argue by contradiction. Suppose $\left\{x^{r}\right\}$ is unbounded. Then there must exist an arc $j$ and a subsequence $R$ such that $\left|x_{j}^{r}\right| \rightarrow+\infty$ as $r \rightarrow \infty, r \in R$. Since $\left\{d\left(p^{r}\right)\right\}$ is bounded it follows (passing into another subsequence if necessary) that there exists a directed cycle $Y$ such that $x_{j}^{r} \rightarrow+\infty$ for all $j \in Y^{+}$, and $x_{j}^{r} \rightarrow-\infty$ for all $j \in Y^{-}$as $r \rightarrow \infty, r \in R$. Since by the CS condition (17)

$$
f_{j}^{-}\left(x_{j}^{r}\right) \leqq t_{j}^{r} \leqq f_{j}^{+}\left(x_{j}^{r}\right),
$$

and also

$$
\sum_{j \in Y^{+}} t_{j}^{r}-\sum_{j \in Y^{-}} t_{j}^{r}=0
$$

we have for all $r$

$$
\sum_{j \in Y^{+}} f_{j}^{-}\left(x_{j}^{r}\right)-\sum_{j \in Y^{-}} f_{j}^{+}\left(x_{j}^{r}\right) \leqq 0 .
$$

This is a contradiction since $x_{j}^{r} \rightarrow+\infty$ implies $f_{j}^{-}\left(x_{j}^{r}\right) \rightarrow+\infty$ while $x_{j}^{r} \rightarrow-\infty$ implies $f_{j}^{+}\left(x_{j}^{r}\right) \rightarrow-\infty$. QED

The next result is remarkable in that it shows that under a mild restriction on the way the relaxation iteration is carried out (which is typically very easy to satisfy in practice), the sequence of price vectors approaches the dual optimal set in an unusual manner. The result depends on the monotonicity of the functions $\nabla g_{j}$. 
We now define a Gauss-Seidel type of algorithm similar to the one sketched in $\S 1$ whereby at each iteration a node $s$ with positive (negative) deficit $d_{s}(p)$ is chosen and $p_{s}$ is decreased (increased) with the aim of decreasing the dual cost $q(p)$. More formally, we initially choose a price vector $p$ and a fixed scalar $\delta$ in the interval $(0,1)$. Then we execute repeatedly the relaxation iteration described below.

Relaxation iteration for strictly convex arc costs.

If $d_{i}(p)=0 \forall i \in N$ then STOP.

Else

Choose any node $s$. Set $\beta=d_{s}(p)$.

If $\beta=0$, do nothing.

If $\beta>0$, then decrease $p_{s}$ so that $0 \leqq d_{s}(p) \leqq \delta \beta$.

If $\beta<0$, then increase $p_{s}$ so that $0 \geqq d_{s}(p) \geqq \delta \beta$.

The only assumption we make regarding the order in which nodes are chosen for relaxation is the following.

Assumption C. Every node in $N$ is chosen as the node $s$ in the relaxation iteration an infinite number of times.

The relaxation iteration is well defined, in the sense that every step in the iteration is executable. To see this suppose that $\beta>0$ and there does not exist a $\Delta<0$ such that $d_{s}\left(p+\Delta e_{s}\right) \leqq \delta \beta$, where $e_{s}$ denotes the $s$ th coordinate vector. Then using the definition of $d, l_{j}$, and $c_{j}$, it is easily seen that

$$
\lim _{\Delta \rightarrow-\infty} d_{s}\left(p+\Delta e_{s}\right)=\sum_{e_{s j}>0} e_{s j} l_{j}+\sum_{e_{s j}<0} e_{s j} c_{j} \geqq \delta \beta>0,
$$

which implies that the flow deficit of node $s$ is positive for any flow $x$ within the upper and lower arc capacity bounds and contradicts the existence of a feasible flow (Assumption A). An analogous argument can be made for the case where $\beta<0$.

In order to obtain our convergence result we must show that the sequence of flow vectors generated by the relaxation algorithm approaches the circulation subspace $C$ (given by (5)). The line of argument that we will use is as follows: We will lower bound the amount of improvement in the dual functional $q$ per iteration by a positive quantity. We will then show that if the sequence of flow vectors do not approach the circulation subspace, the quantity itself can be lower bounded by a positive constant which implies that the optimal dual functional has a value of $-\infty$. This will contradict the finiteness of the optimal primal cost.

We will denote the price vector generated at the $r$ th iteration by $p^{r}, r=0,1,2, \cdots$ and the node operated on at the $r$ th iteration by $s^{r}, r=0,1,2, \cdots$. To simplify notation we will denote

$$
t^{r}=E^{T} p^{r}, \quad x_{j}^{r}=\nabla g_{j}\left(t_{j}^{r}\right) .
$$

We denote by $x^{r}$ the vector with coordinates $x_{j}^{r}, j \in A$. Note the symmetry following from the $C S$ condition (16) or (17): $x_{j}^{r}$ is the gradient of the dual cost $g_{j}$ at $t_{j}^{r}$, while $t_{j}^{r}$ is a subgradient of the primal cost $f_{j}$ at $x_{j}^{r}$. For any directed cycle $Y$ of the network we will use $Y^{+}$to denote the set of arcs $\{j \in A \mid j$ is positively oriented in $Y\}$, and $Y^{-}$to denote $Y \backslash Y^{+}$. We first show three preliminary results.

Proposition 2.1. We have for all $r$ such that $p^{r+1} \neq p^{r}$ [i.e. $d_{s^{\prime}}\left(p^{r}\right) \neq 0$ ]

$$
q\left(p^{r}\right)-q\left(p^{r+1}\right) \geqq \sum_{j \in A}\left[f_{j}\left(x_{j}^{r+1}\right)-f_{j}\left(x_{j}^{r}\right)-\left(x_{j}^{r+1}-x_{j}^{r}\right) t_{j}^{r}\right]>0,
$$

with equality holding if line minimization is used $\left[d_{s^{\prime}}\left(p^{r+1}\right)=0\right]$. 
Proposition 2.3. Given $p \in R^{|N|}$, let $s$ be a node and let $\bar{p}$ denote a dual price vector obtained by applying the relaxation iteration to $p$ using node $s$. Assume in addition that $\bar{p}$ is chosen so that

$$
\begin{array}{ll}
\text { if } d_{s}(p)>0 \text { then } d_{s}[\bar{p}+\alpha(p-\bar{p})]>0 & \forall \alpha>0, \\
\text { if } d_{s}(p)<0 \text { then } d_{s}[\bar{p}+\alpha(p-\bar{p})]<0 & \forall \alpha>0 .
\end{array}
$$

Then for all $k \in N$, and all optimal dual price vectors $p^{*}$ we have

$$
\min \left\{p_{i}-p_{i}^{*} \mid i \in N\right\} \leqq \bar{p}_{k}-p_{k}^{*} \leqq \max \left\{p_{i}-p_{i}^{*} \mid i \in N\right\} .
$$

Note. Assumption (19) when $d_{s}(p)>0\left[d_{s}(p)<0\right]$ is equivalent to assuming that $\bar{p}_{s}$ is chosen greater (less) or equal to the largest (smallest) minimizing point of the dual cost along the $s$ th coordinate starting from $p$. It is automatically satisfied if the dual cost has a unique minimizing point along the line $\left\{p+\alpha e_{s} \mid \alpha \in R\right\}$.

Proof. Fix an optimal dual price vector $p^{*}$ and consider an arbitrary price vector $\tilde{p}$. Let $k$ be such that $\tilde{p}_{k}-p_{k}^{*}=\max \left\{\tilde{p}_{i}-p_{i}^{*} \mid i \in N\right\}$. We have

$$
\tilde{p}_{k}-p_{k}^{*} \geqq \tilde{p}_{i}-p_{i}^{*} \quad \forall i \neq k
$$

so that

$$
\tilde{p}_{k}-\tilde{p}_{i} \geqq p_{k}^{*}-p_{i}^{*} \quad \forall j \sim(k, i), \quad \tilde{p}_{i}-\tilde{p}_{k} \leqq p_{i}^{*}-p_{k}^{*} \quad \forall j \sim(i, k) .
$$

Since $\nabla g_{j}$ is a nondecreasing function, we have that

$$
\nabla g_{j}\left(\tilde{p}_{k}-\tilde{p}_{i}\right) \geqq \nabla g_{j}\left(p_{k}^{*}-p_{i}^{*}\right) \quad \forall j \sim(k, i), \quad \nabla g_{j}\left(\tilde{p}_{i}-\tilde{p}_{k}\right) \leqq \nabla g_{j}\left(p_{i}^{*}-p_{k}^{*}\right) \quad \forall j \sim(i, k) \text {. }
$$

Thus $d_{k}(\tilde{p}) \geqq d_{k}\left(p^{*}\right)=0$.

The desired assertion (20) holds if $d_{s}(p)=0$ since then we have $\bar{p}=p$. Assume that $d_{s}(p)<0$. Consider the vector $\tilde{p}$ defined by

$$
\tilde{p}_{i}=\left\{\begin{array}{l}
p_{i} \text { if } i \neq s, \\
p_{s}^{*}+\max \left\{p_{j}-p_{j}^{*} \mid j \in N\right\} \quad \text { if } i=s .
\end{array}\right.
$$

Then we have $\tilde{p}_{s}-p_{s}^{*}=\max \left\{\tilde{p}_{i}-p_{i}^{*} \mid i \in N\right\}=\max \left\{p_{i}-p_{i}^{*} \mid i \in N\right\}$ and by the preceding argument we have $d_{s}(\tilde{p}) \geqq 0$. Therefore, using assumption (19), we have $\bar{p}_{s} \leqq \tilde{p}_{s}$ while at the same time $p_{s}<\bar{p}_{s}$, and $p_{i}=\bar{p}_{i}$ for all $i \neq s$. The assertion (20) follows. The proof is similar when $d_{s}(p)>0$. Q.E.D.

Note that Proposition 2.3 implies among other things that, if (19) is satisfied at all iterations, the sequence $\left\{p^{r}\right\}$ generated by the relaxation method is bounded. Furthermore if we can show that $\left\{p^{r}\right\}$ accumulates at an optimal price vector, the proposition implies that $\left\{p^{\prime}\right\}$ must converge to that vector. We are now ready to show our main result.

Proposition 2.4. Let $\left\{p^{r}, x^{r}\right\}$ be a sequence generated by the relaxation method for strictly convex arc costs. Then

$$
\begin{aligned}
& \text { (a) } \lim _{r \rightarrow \infty} d\left(p^{r}\right)=0 . \\
& \text { (b) } \lim _{r \rightarrow \infty} x^{r}=x^{*}
\end{aligned}
$$

where $x^{*}$ is the unique optimal flow vector.

(c) $\lim _{r \rightarrow \infty} q\left(p^{r}\right)=-f\left(x^{*}\right)=\inf _{p} q(p)$. 
(d) If condition (19) is satisfied at each iteration, and the dual problem has an optimal solution, then

$$
\lim _{r \rightarrow \infty} p^{r} \rightarrow p^{*}
$$

where $p^{*}$ is some optimal price vector.

Proof. (a) We first show that

$$
\lim _{r \rightarrow \infty} d_{s^{r}}\left(p^{r}\right)=0
$$

Indeed, if this is not so there must exist an $\varepsilon>0$ and a subsequence $R$ such that $\left|d_{s^{\prime}}\left(p^{r}\right)\right| \geqq \varepsilon$ for all $r \in R$. Without loss of generality we assume that $d_{s^{r}}\left(p^{r}\right) \geqq \varepsilon$ for all $r \in R$. Since $\delta\left|d_{s^{\prime}}\left(p^{r}\right)\right| \geqq\left|d_{s^{\prime}}\left(p^{r+1}\right)\right|$ we have that at the $r$ th iteration some arc incident to node $s^{r}$ must change its flow by at least $\Delta$ where $\Delta=(1-\delta) \varepsilon /|A|$. By passing to a subsequence if necessary we assume that this happens for the same arc $j^{*}$ for all $r \in R$, and that $x_{j^{*}}^{r+1}-x_{j^{*}}^{r} \geqq \Delta$, for all $r \in R$. Using the boundedness of $\left\{x^{r}\right\}$ (Proposition 2.2) we may also assume that the subsequence $\left\{x_{j^{*}}^{r}\right\}_{r \in R}$ converges to some $x_{j^{*}}$. Using the convexity of $f_{j}$ and Proposition 2.1 we have

$$
\begin{aligned}
q\left(p^{r}\right)-q\left(p^{r+1}\right) & \geqq f_{j^{*}}\left(x_{j^{*}}^{r+1}\right)-f_{j^{*}}\left(x_{j^{*}}^{r}\right)-\left(x_{j^{*}}^{r+1}-x_{j^{*}}^{r}\right) t_{j^{*}}^{r} \\
& \geqq f_{j^{*}}\left(x_{j^{*}}^{r}+\Delta\right)-f_{j^{*}}\left(x_{j^{*}}^{r}\right)-\Delta t_{j^{*}}^{r} \\
& \geqq f_{j^{*}}\left(x_{j^{*}}^{r}+\Delta\right)-f_{j^{*}}\left(x_{j^{*}}^{r}\right)-\Delta f_{j^{*}}^{+}\left(x_{j^{*}}^{r}\right.
\end{aligned}
$$

Taking the limit as $r \rightarrow \infty, r \in R$ and using the facts $x_{j^{*}}^{r} \rightarrow x_{j^{*}}$ and $\lim _{r \rightarrow \infty} f_{j^{*}}^{+}\left(x_{j^{*}}^{r}\right) \leqq f_{j^{*}}^{+}\left(x_{j^{*}}\right)$ (in view of the upper semicontinuity of $f_{j^{*}}^{+}$) we obtain

$$
\underset{\substack{r \rightarrow \infty \\ r \in R}}{\liminf }\left[q\left(p^{r}\right)-q\left(p^{r+1}\right)\right] \geqq f_{j^{*}}\left(x_{j^{*}}+\Delta\right)-f_{j^{*}}\left(x_{j^{*}}\right)-\Delta f_{j^{*}}^{+}\left(x_{j^{*}}\right)>0 .
$$

This implies that $\lim _{r \rightarrow \infty} q\left(p^{r}\right)=-\infty$. But this is not possible because from (6) and (12) we have $q(p) \geqq-\sum_{j \in A} f_{j}\left(x_{j}\right)$ for all $p$ and $x \in C$. Therefore (24) is proved by contradiction.

We now show (21). Choose any $i \in N$. Take any $\varepsilon>0$ and let $R$ be the set of indices $r$ such that $d_{i}\left(p^{r}\right)>2 \varepsilon$. Assume without loss of generality that $d_{i}\left(p^{r}\right)<\varepsilon$ for all $r$ with $i=s^{r}$ (cf. (24)). For every $r \in R$ let $r^{\prime}$ be the first index with $r^{\prime}>r$ such that $i=s^{r^{\prime}}$. Then during iterations $r, r+1, \cdots, r^{\prime}-1$ node $i$ is not chosen for relaxation while its deficit decreases from greater than $2 \varepsilon$ to lower than $\varepsilon$. We claim that during these iterations the total deficit $\sum_{k \in N}\left|d_{k}(p)\right|$ is decreased by an amount of more than $2 \varepsilon$. To see this, note that the total absolute deficit cannot increase at any iteration as noted earlier in the proof of Proposition 2.2. Next observe that for any of the iterations $r, r+1, \cdots, r^{\prime}-1$, say $\bar{r}$, for which the deficit of $i$ is decreased by an amount $\xi>0$ from a positive value $d_{i}\left(p^{\bar{r}}\right)>0$, it must be that the node $s$ chosen for relaxation is a neighbor of $i$ and has a negative deficit $d_{s}\left(p^{r}\right)<0$. Since all increase in $d_{s}\left(p^{r}\right)$ during the iteration must be matched by decreases of the deficits of the neighbor nodes of $s$, and the deficit of $s$ will remain nonpositive after the iteration, it follows that the total absolute deficit will be decreased by at least $2 \min \left\{\xi, d_{i}\left(p^{r}\right)\right\}$ during the iteration. This shows that during iterations $r, r+1, \cdots, r^{\prime}-1$ the total absolute deficit must decrease by more than $2 \varepsilon$. It follows that the set $R$ of indices $r$ for which $d_{i}\left(p^{r}\right)>2 \varepsilon$ cannot be infinite. Since $\varepsilon>0$ is arbitrary we obtain $\lim \sup _{r \rightarrow \infty} d_{i}\left(p^{r}\right) \leqq 0$. Similarly we can show that $\lim _{\inf } \rightarrow \infty d_{i}\left(p^{r}\right) \geqq 0$ and therefore $d_{i}\left(p^{r}\right) \rightarrow 0$. 
(b) For all $r$ and arcs $j$ we have the CS condition

$$
f_{j}^{-}\left(x_{j}^{r}\right) \leqq t_{j}^{r} \leqq f_{j}^{+}\left(x_{j}^{r}\right) \text {. }
$$

If $Y$ is any cycle we have

$$
\sum_{j \in Y^{+}} t_{j}^{r}-\sum_{j \in Y^{-}} t_{j}^{r}=0
$$

so from (25) we obtain

$$
\sum_{j \in Y^{+}} f_{j}^{-}\left(x_{j}^{r}\right)-\sum_{j \in Y^{-}} f_{j}^{+}\left(x_{j}^{r}\right) \leqq 0 \leqq \sum_{j \in Y^{+}} f_{j}^{+}\left(x_{j}^{r}\right)-\sum_{j \in Y^{-}} f_{j}^{-}\left(x_{j}^{r}\right)
$$

Let $\left\{x^{r}\right\}_{r \in R}$ be a subsequence converging to some $\bar{x}$ (cf. Proposition 2.2). Then from (26) and the lower (upper) semicontinuity of $f_{j}^{-}\left(f_{j}^{+}\right)$, we have for all cycles $Y$

$$
\sum_{j \in Y^{+}} f_{j}^{-}\left(\bar{x}_{j}\right)-\sum_{j \in Y^{-}} f_{j}^{+}\left(\bar{x}_{j}\right) \leqq 0 \leqq \sum_{j \in Y^{+}} f_{j}^{+}\left(\bar{x}_{j}\right)-\sum_{j \in Y^{-}} f_{j}^{-}\left(\bar{x}_{j}\right),
$$

while from part (a) we have $\bar{x} \in C$. This implies that $\bar{x}$ is an optimal flow ([3, Chap. $8]$ ) and therefore must be equal to the unique optimal flow $x^{*}$. Since, by Proposition 2.2, $\left\{x^{r}\right\}$ is bounded we obtain $x^{r} \rightarrow x^{*}$.

(c) For every arc $j$ for which $l_{j}<c_{j}$ there are three possibilities:

(1) $\left\{t_{j}^{r}\right\}$ is bounded.

(2) $x_{j}^{*}=c_{j}<+\infty, x_{j}^{r} \leqq x_{j}^{*}$ and $-\infty<\liminf _{r \rightarrow \infty} t_{j}^{r} \leqq \lim \sup _{r \rightarrow \infty} t_{j}^{r}=+\infty$.

(3) $x_{j}^{*}=l_{j}>-\infty, x_{j}^{r} \geqq x_{j}^{*}$ and $-\infty=\lim \inf _{r \rightarrow \infty} t_{j}^{r} \leqq \lim \sup _{r \rightarrow \infty} t_{j}^{r}<+\infty$, while for an arc $j$ with $l_{j}=c_{j}$ we must have $x_{j}^{*}=x_{j}^{\prime}$ for all $r$. Using this fact we can easily see that we can construct a subsequence $R$ such that

$$
\sum_{j \in A} t_{j}^{r}\left(x_{j}^{r}-x_{j}^{*}\right) \leqq \sum_{j \in B} t_{j}^{r}\left(x_{j}^{r}-x_{j}^{*}\right) \quad \forall r \in R
$$

where $B$ is a set of arcs $j$ such that $\left\{t_{j}^{r}\right\}_{R}$ is bounded. We have (since $t^{r} \in C^{\perp}, x^{*} \in \dot{C}$, and therefore $\left.\sum_{j \in A} t_{j}^{r} x_{j}^{*}=0\right)$

$$
f\left(x^{r}\right)+q\left(p^{r}\right)=\sum_{j \in A} t_{j}^{r} x_{j}^{r}=\sum_{j \in A} t_{j}^{r}\left(x_{j}^{r}-x_{j}^{*}\right) \leqq \sum_{j \in B} t_{j}^{r}\left(x_{j}^{r}-x_{j}^{*}\right) .
$$

Since $x_{j}^{r} \rightarrow x_{j}^{*}$ and $\left\{t_{j}^{r}\right\}_{R}$ is bounded for $j \in B$ we obtain by taking the limit above $f\left(x^{*}\right)+\lim _{r \rightarrow \infty} q\left(p^{r}\right) \leqq 0$. On the other hand we have for all $p$ using (6) and (12) $f\left(x^{*}\right)+q(p) \geqq 0$. This together with the preceding relation show the desired result.

(d) By Proposition 2.3, $\left\{p^{r}\right\}$ is bounded. Let $\left\{p^{r}\right\}_{r \in R}$ be a subsequence converging to a vector $p^{*}$ and let $t^{*}=E^{T} p^{*}$. We have for all $j \in A$

$$
f_{j}^{-}\left(x_{j}^{r}\right) \leqq t_{j}^{r} \leqq f_{j}^{+}\left(x_{j}^{r}\right) \quad \forall r \in R .
$$

It follows using part (b) and the lower (upper) semicontinuity of $f_{j}^{-}\left(f_{j}^{+}\right)$that for all $j \in A, f_{j}^{-}\left(x_{j}^{*}\right) \leqq t_{j}^{*} \leqq f_{j}^{+}\left(x_{j}^{*}\right)$ where $x^{*}$ is the optimal flow vector. Therefore $t^{*}$ satisfies together with $x^{*}$ the complementary slackness conditions and must be dual optimal. Proposition 2.3 shows that $\left\{p^{\prime}\right\}$ cannot have two different dual optimal price vectors as limit points and the conclusion follows. QED

3. The relaxation method for mixed linear and strictly convex arc costs. We first introduce some terminology. We will say that a point $b \in \operatorname{dom}\left(f_{j}\right)$ is a breakpoint of $f_{j}$ if $f_{j}^{-}(b)<f_{j}^{+}(b)$. Note that the dual functional $q$, as given by (12), is separable and is piecewise either linear or strictly convex. Roughly speaking each linear piece (breakpoint) of the primal cost function $f_{j}$ corresponds to a breakpoint (linear piece) of the dual cost function $g_{j}$ (see Fig. 3.1). 

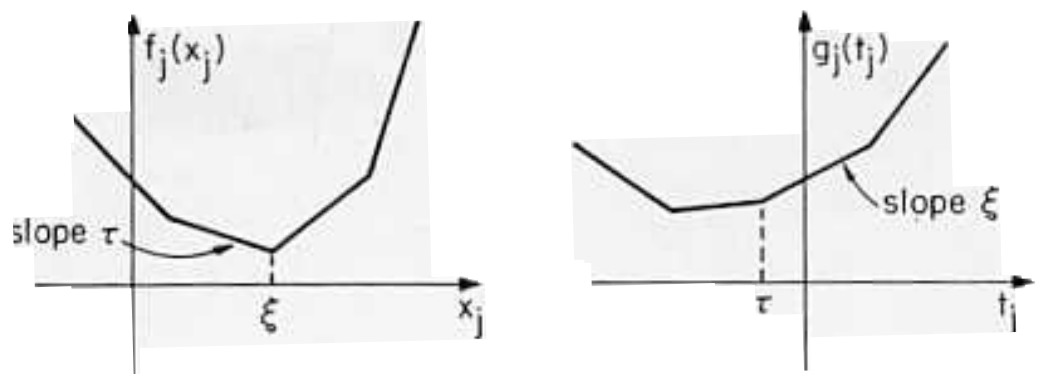

FIG. 3.1. Correspondence between the breakpoints of $f_{j}$ and the linear pieces of $g_{j}$ (and vice versa).

Assumption D. $f_{j}^{+}\left(x_{j}\right)>-\infty$ and $f_{j}^{-}\left(x_{j}\right)<+\infty$ for all $x_{j} \in \operatorname{dom}\left(f_{j}\right)$.

In the terminology of ([3, Chap. 8]), Assumption D implies that every feasible primal solution is regularly feasible and guarantees, together with Assumptions $A$ and $B$, that the dual problem has an optimal solution $([3$, p. 360]). For a given $\varepsilon>0$, we say that $x \in R^{|A|}$ and $p \in R^{|N|}$ satisfy $\varepsilon$-Complementary Slackness ( $\varepsilon$-CS for short) if

$$
f_{j}^{-}\left(x_{j}\right)-\varepsilon \leqq t_{j} \leqq f_{j}^{+}\left(x_{j}\right)+\varepsilon \quad \forall j \in A
$$

where $t=E^{T} p$. For a given $p,(27)$ defines upper and lower bounds, called $\varepsilon$-bounds, on the flow vector:

$$
l_{j}^{\varepsilon}=\min \left\{\xi \mid f_{j}^{+}(\xi) \geqq t_{j}-\varepsilon\right\}, \quad c_{j}^{\varepsilon}=\max \left\{\xi \mid f_{j}^{-}(\xi) \leqq t_{j}+\varepsilon\right\} \quad \forall j \in A .
$$

Then $x$ and $p$ satisfying $\varepsilon$-CS is equivalent to

$$
x_{j} \in\left[l_{j}^{\varepsilon}, c_{j}^{\varepsilon}\right] \quad \forall j \in A
$$

where $t=E^{T}$. For a given $t_{j}$, we can obtain $l_{j}^{\varepsilon}$ and $c_{j}^{\varepsilon}$ from the graph of the subdifferential mapping of $f_{j}$ as shown in Figs. 3.2-3.3. Intuition suggests that if $x$ is in the

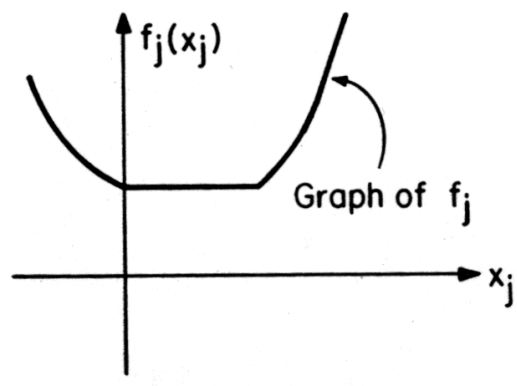

FIG. 3.2. Graph of $f_{j}$.

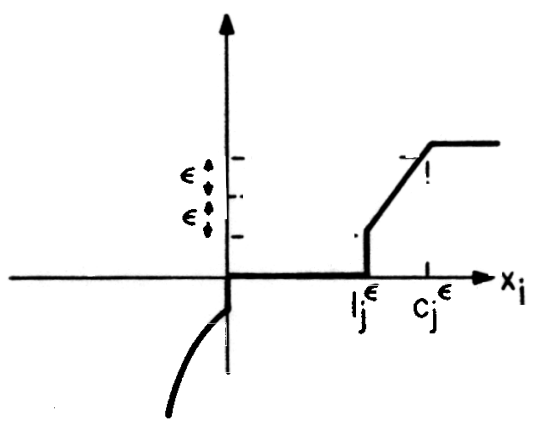

Fig. 3.3. Graph of $\partial f_{j}$ and $\varepsilon$-bounds corresponding to $t_{j}$. 
circulation subspace $C, x$ and $p$ satisfy $\varepsilon$-CS and $\varepsilon$ is small, then both $x$ and $p$ should be near optimal. This idea will be made precise later when we explore the near optimality properties of the solution generated by a relaxation algorithm that uses the notion of $\varepsilon$-CS. The definition of $\varepsilon$-CS is related to the $\varepsilon$-subgradient idea introduced in nondifferentiable optimization in [13] as well as to the fortified descent method of Rockafellar [3]. The latter method, however, for a given $p$ and $t=E^{T} p$, uses different lower and upper bounds on $x_{j}$ given by

$$
\inf _{\Delta>0} \frac{g_{j}\left(t_{j}+\Delta\right)-g_{j}\left(t_{j}\right)+\varepsilon}{\Delta} \text { and } \sup _{\Delta>0} \frac{g_{j}\left(t_{j}\right)-g_{j}\left(t_{j}-\Delta\right)-\varepsilon}{\Delta} .
$$

Our bounds of (28) seem simpler for implementation purposes particularly when some of the cost functions $f_{j}$ are linear within their effective domain.

For a given $x$ within the $\varepsilon$-bounds, we define the deficit of node $i$ as in (2) and say that a sequence of nodes $\left\{n_{1} \cdots n_{k}\right\}$ forms a flow augmenting path if

$$
d_{n_{1}}<0, d_{n_{k}}>0 \text { and }\left\{\begin{array}{lll}
x_{j}<c_{j}^{\varepsilon} & \text { if } j \sim\left(n_{m}, n_{m+1}\right), & m \in\{1, \cdots, k-1\}, \\
x_{j}>l_{j}^{\varepsilon} & \text { if } j \sim\left(n_{m+1}, n_{m}\right), & m \in\{1, \cdots, k-1\} .
\end{array}\right.
$$

Let

$$
\mu_{m}=\left\{\begin{array}{ll}
c_{j}^{\varepsilon}-x_{j} & \text { if } j \sim\left(n_{m}, n_{m+1}\right), \\
x_{j}-l_{j}^{\varepsilon} & \text { if } j \sim\left(n_{m+1}, n_{m}\right),
\end{array} \quad m \in\{1, \quad, k \quad 1\}\right.
$$

We will call

$$
\mu=\min \left\{-d_{n_{1}}, d_{n_{k}}, \mu_{1}, \cdots, \mu_{k-1}\right\}
$$

the capacity of the path. The relaxation algorithm of this section uses the labeling method of Ford and Fulkerson [14] for finding flow augmenting paths and for augmenting flow along them.

For a given tension vector $t \in C$ and any subset of nodes $S$, we define $C_{\varepsilon}(S, t)$ by

where we use the notation

$$
C_{\varepsilon}(S, t)=\sum_{j \in[S, N \backslash S]} l_{j}^{\varepsilon}-\sum_{j \in[N \backslash S, S]} c_{j}^{\varepsilon}
$$

$$
[S, N \backslash S]=\{j \mid j \sim(i, k), i \in S, k \notin S\}, \quad N \backslash S, S]=\{j \mid j \sim(i, k), i \notin S, k \in S\} .
$$

We also define the $|N|$-vector $u(S)$ by

$$
u_{i}(S)= \begin{cases}-1 & \text { if } i \in S, \\ 0 & \text { if } i \notin S .\end{cases}
$$

The importance of these notions is due to the fact that for any $\varepsilon \geqq 0, C_{\varepsilon}(S, t)>0$ implies that $u(S)$ is a dual descent direction at $p$, where $p$ is any price vector satisfying $E^{T} p=t$. This follows from the fact that the directional derivative of $q$ at $p$ in the direction $u(S)$ defined by

$$
q^{\prime}(p ; u(S))=\lim _{\Delta \downarrow 0} \frac{q(p+\Delta u(S))-q(p)}{\Delta}
$$

is easily verified to be

$$
q^{\prime}(p ; u(S))=\sum_{j \in[N \backslash S, S]} c_{j}^{0}-\sum_{j \in[S, N \backslash S]} l_{j}^{0} \leqq-C_{\varepsilon}(S, t)
$$

where $c_{j}^{0}, l_{j}^{0}$ are the $\varepsilon$-bounds corresponding to $\varepsilon=0$, and we are making use of the fact $c_{j}^{0} \leqq c_{j}^{\varepsilon}, l_{j}^{0} \geqq l_{j}^{\varepsilon}$, for all $\varepsilon \geqq 0$.

We now describe the relaxation algorithm. The algorithm is iterative and uses the $\varepsilon$-CS idea. The scalar $\varepsilon$ is kept fixed throughout the algorithm. At the beginning of 
each iteration we have a dual price vector $p$ and a flow vector $x$ satisfying $l_{j}^{\varepsilon} \leqq x_{j} \leqq c_{j}^{\varepsilon}$ for all $j \in A$. If $x \in C$ then we terminate. Otherwise we use labeling to either find a flow augmenting path, in which case a flow augmentation is performed to bring $x$ "closer" to $C$; or to find a dual descent direction, in which case a dual descent along this direction is performed. When each $f_{j}$ is linear within its effective domain, $\varepsilon=0$, and all problem data is integer, the algorithm coincides with the relaxation method of [1], [2]. When each $f_{j}$ is strictly convex and $\varepsilon=0$ the algorithm coincides with the exact line minimization version of the algorithm of the previous section $(\delta=0)$.

\section{Relaxation iteration.}

Step 0 . Given $p$ and $x$ satisfying $l_{j}^{\varepsilon} \leqq x_{j} \leqq c_{j}^{\varepsilon}$ for all $j$, let $t$ and $d$ be the corresponding tension and deficit vectors.

Step 1. Pick a node $s$ such that $d_{s}>0$. If no such node exists terminate. Else set all nodes to be unlabeled and unscanned. Give the label 0 to node $s$. Set $S=\varnothing$ and go to Step 2.

Step 2. Choose a labeled but unscanned node $k$. Set $S \leftarrow S \cup\{k\}$ and go to Step 3 .

Step 3. Scan the label of the node $k$ as follows: Give the label $k$ to all unlabeled nodes $m$ such that $x_{j}<c_{j}^{\varepsilon}$ for $j \sim(m, k)$ and to all unlabeled nodes $m$ such that $x_{j}>l_{j}^{\varepsilon}$ for $j \sim(k, m)$. If $C_{\varepsilon}(S, t)>0$ then go to Step 5. Else if for any of the nodes $m$ labeled from $k$ we have $d_{m}<0$ then go to Step 4. Else go to Step 2.

Step 4. (Flow Augmentation Step). A flow augmenting path has been found which starts at the node $m$ (with $d_{m}<0$ ) identified in Step 3 and ends at the node $s$. The path can be constructed by tracing labels starting from $m$. Let $\mu$ be the capacity of the path. Increase by $\mu$ the flow of all arcs on the path oriented in the direction from $m$ to $s$, decrease by $\mu$ the flow of all other arcs on the path. Update the deficit vector $d$ and return.

Step 5. (Dual Descent Step). Determine $\lambda^{*}$ such that

$$
q\left(p+\lambda^{*} u(S)\right)=\min _{i \rightarrow n}\{q(p+\lambda u(S))\}
$$

Set $p \leftarrow p+\lambda^{*} u(S)$ and update the bounds $l_{j}^{\varepsilon}$ and $c_{j}^{\varepsilon}$. Update $x$ to maintain the $\varepsilon$-CS condition $l_{j}^{\varepsilon} \leqq x_{j} \leqq c_{j}^{\epsilon}$ and return.

Validity and finite termination of the relaxation iteration. We will show that, under Assumption D, all steps in the Relaxation Iteration are executable and that the iteration terminates in a finite number of operations.

Steps 0,1 , and 3 are trivially executable. Step 2 is certainly executable on its first pass since the node $s$ is labeled but unscanned. To show that it remains executable on subsequent passes we only need to verify that each time we go to Step 2 from Step 3 there always exists a labeled but unscanned node. In Step 2, if all labeled nodes are also scanned we have

$$
\begin{aligned}
\sum_{i \in S} d_{i} & =\sum_{j \in[S, N \backslash S]} x_{j}-\sum_{j \in[N \backslash S, S]} x_{j} \\
& =\sum_{j \in[S, N \backslash S]} l_{j}^{\varepsilon}-\sum_{j \in[N \backslash S, S]} c_{j}^{\varepsilon}=C_{\varepsilon}(S, t) .
\end{aligned}
$$

Since node $s$ has positive deficit and all other labeled nodes have nonnegative deficits we obtain that $C_{\varepsilon}(S, t)>0$ and therefore in the previous pass through Step 3 we would have branched to Step 5 rather than to Step 2. Step 4 is executable since the rule for 
labeling ensures that a flow augmenting path exists from node $m$ to node $s$, so a flow augmentation is possible. Step 5 is executable since $C_{\varepsilon}(S, t)>0$ implies that $u(S)$ is a dual descent direction at $p$, and we can show that there exists a minimizing stepsize $\lambda^{*}$. To see this assume the contrary, i.e., that there does not exist a stepsize $\lambda^{*}$ achieving the minimum along the direction $u(S)$. In that case the convexity of $q$ implies that

$$
\begin{aligned}
& q^{\prime}(p+\lambda u(S) ; u(S))<0 \quad \forall \lambda>0, \\
& \lim _{\lambda \rightarrow+\infty} q^{\prime}(p+\lambda u(S) ; u(S)) \leqq 0 .
\end{aligned}
$$

Then it can be easily seen that either

$$
\sum_{j \in[S, N \backslash S]} l_{j}-\sum_{j \in[N \backslash S, S]}
$$

in which case Assumption A is violated [dom $(f) \cap C$ is empty], or

$$
\sum_{j \in[S, N \backslash S]} l_{j}-\sum_{j \in[N \backslash S, S]} c_{j}=0
$$

and either $f_{j}^{+}\left(l_{j}\right)=-\infty$ for some $j \in[S, N / S]$ or $f_{j}^{-}\left(c_{j}\right)=+\infty$ for some $j \in[N \backslash S, S]$, in which case Assumption $D$ is violated. To complete the proof that the relaxation iteration terminates in a finite number of operations we note that we cannot loop between Step 2 and Step 3 infinitely often since the number of scanned nodes is increased by one each time we visit Step 3.

We next show that the relaxation algorithm, when applied in conjunction with an easily implementable labeling rule, terminates in a finite number of iterations. The proof may be divided into two separate parts. The first part involves showing that the number of dual descent steps is not infinite. This is done by arguing that the optimal dual cost is necessarily $-\infty$ if the number of dual descent steps is infinite. The second part involves showing that the number of flow augmentations between successive dual descent steps is finite. This is done by choosing an appropriate labeling scheme for the relaxation algorithm and showing that the number of flow augmentations is finite under the chosen scheme. For this purpose we will propose two schemes: breadth-first search and arc discrimination.

We first show that the stepsize in each dual descent step is bounded from below by $\varepsilon$. Indeed our definition of $\varepsilon$-CS was motivated primarily by this fact.

Proposition 3.1. The stepsize in each dual descent step is greater than $\varepsilon$.

Proof. Under Assumption B, $q(p)$ is subdifferentiable everywhere. Let $S$ denote the subset of nodes corresponding to the dual descent direction generated by the relaxation iteration. In other words, the dual descent direction $u$ is given by

$$
u_{i}= \begin{cases}-1 & \text { if } i \in S, \\ 0 & \text { if } i \notin S,\end{cases}
$$

and $S$ satisfies $C_{\varepsilon}(S, t)>0$. Now consider $p^{\prime}$ given by $p^{\prime}=p+\varepsilon u$ and $t^{\prime}=E^{T} p^{\prime}$. Then

$$
\begin{array}{ll}
t_{j}^{\prime}=t_{j}-\varepsilon & \text { if } j \in[S, N \backslash S], \\
t_{j}^{\prime}=t_{j}+\varepsilon & \text { if } j \in[N \backslash S, S], \\
t_{j}^{\prime}=t_{j} & \text { otherwise, }
\end{array}
$$


so that

$$
\begin{aligned}
& l_{j}^{\varepsilon}=\min \left\{\xi \mid f_{j}^{+}(\xi) \geqq t_{j}-\varepsilon\right\}=\min \left\{\xi \mid f_{j}^{+}(\xi) \geqq t_{j}^{\prime}\right\} \quad \text { for all } j \in[S, N \backslash S], \\
& c_{j}^{\varepsilon}=\max \left\{\xi \mid f_{j}^{-}(\xi) \leqq t_{j}+\varepsilon\right\}=\max \left\{\xi \mid f_{j}^{-}(\xi) \leqq t_{j}^{\prime}\right\} \quad \text { for all } j \in[N \backslash S, S]
\end{aligned}
$$

Therefore

$$
q^{\prime}\left(p^{\prime} ; u\right)=-\sum_{j \in[S, N \backslash S]} l_{j}^{\varepsilon}+\sum_{j \in[N \backslash S, S]} c_{j}^{\varepsilon}=-C_{\varepsilon}(S, t)<0 .
$$

Since $q$ is convex, $q^{\prime}(p ; u)<0$ and $q^{\prime}(p+\varepsilon u ; u)<0$ imply that $q^{\prime}(p+\alpha u ; u)<0$ for all $\alpha \in[0, \varepsilon]$. Therefore the stepsize in a dual descent step is greater than $\varepsilon$. QED

We will now use Proposition 3.1 to prove that the number of dual descent steps is necessarily finite. The following result is a first step in this direction.

Proposition 3.2. Let $p^{r}$ denote the price vector generated by the relaxation algorithm just before the rth dual descent step. Then for each $r \in\{0,1,2, \cdots\}$

where we define

$$
q\left(p^{r}\right)-q\left(p^{r+1}\right)>\sum_{\substack{j \in\left[S^{r}, N \backslash S_{j}\right] \text { or } \\ j \in\left[N \backslash S^{r}, S^{r}\right]}}\left[f_{j}\left(\Psi_{j}^{r}\right)-f_{j}\left(X_{j}^{r}\right)-\left(\Psi_{j}^{r}-X_{j}^{r}\right) t_{j}^{r}\right] \geqq 0
$$

$$
\Psi_{j}^{r}=\left\{\begin{array}{ll}
g_{j}^{+}\left(t_{j}^{r}-\varepsilon\right) & \text { if } j \in\left[S^{r}, N \backslash S^{r}\right], \\
g_{j}^{-}\left(t_{j}^{r}+\varepsilon\right) & \text { if } j \in\left[N \backslash S^{r}, S^{r}\right],
\end{array} \quad X_{j}^{r}= \begin{cases}g_{j}^{-}\left(t_{j}^{r}\right) & \text { if } j \in\left[S^{r}, N \backslash S^{r}\right], \\
g_{j}^{+}\left(t_{j}^{r}\right) & \text { if } j \in\left[N \backslash S^{r}, S^{r}\right],\end{cases}\right.
$$

and $S^{r}$ denotes the node subset corresponding to the descent direction at the rth dual descent step.

Proof. From the definition of $\Psi_{j}^{r}$ and $X_{j}^{r}$ we have that

$$
\begin{array}{lll}
g_{j}\left(t_{j}^{r}\right)=X_{j}^{r} t_{j}^{r}-f_{j}\left(X_{j}^{r}\right), & g_{j}\left(t_{j}^{r}-\varepsilon\right)=\Psi_{j}^{r}\left(t_{j}^{r}-\varepsilon\right)-f_{j}\left(\Psi_{j}^{r}\right) & \forall j \in\left[S^{r}, N \backslash S^{r}\right], \\
g_{j}\left(t_{j}^{r}\right)=X_{j}^{r} t_{j}^{r}-f_{j}\left(X_{j}^{r}\right), & g_{j}\left(t_{j}^{r}+\varepsilon\right)=\Psi_{j}^{r}\left(t_{j}^{r}+\varepsilon\right)-f_{j}\left(\Psi_{j}^{r}\right) & \forall j \in\left[N \backslash S^{r}, S^{r}\right] .
\end{array}
$$

From the definition of $q, S^{r}$ and $u\left(S^{r}\right)$ we have that

$$
\begin{aligned}
q\left(p^{r}+\varepsilon u\left(S^{r}\right)\right)=q\left(p^{r}\right) & +\sum_{j \in\left[S^{\prime}, N \backslash s^{r}\right]}\left[g_{j}\left(t_{j}^{r}-\varepsilon\right)-g_{j}\left(t_{j}^{r}\right)\right] \\
& +\sum_{j \in\left[N \backslash S^{r}, s^{r}\right]}\left[g_{j}\left(t_{j}^{r}+\varepsilon\right)-g_{j}\left(t_{j}^{r}\right)\right]
\end{aligned}
$$

and from Proposition 3.1 we have that

$$
q\left(p^{r}\right)-q\left(p^{r+1}\right) \geqq q\left(p^{r}\right)-q\left(p^{r}+\varepsilon u\left(S^{r}\right)\right) .
$$

Combining the above three sets of equalities and inequalities we obtain that

$$
\begin{aligned}
q\left(p^{r}\right)-q\left(p^{r+1}\right) \geqq & \sum_{j \in\left[s^{\prime}, N \backslash s^{r}\right]}\left[\left[X_{j}^{r} t_{j}^{r}-f_{j}\left(X_{j}^{r}\right)\right]-\left[\left(t_{j}^{r}-\varepsilon\right) \Psi_{j}^{r}-f_{j}\left(\Psi_{j}^{r}\right)\right]\right] \\
& +\sum_{j \in\left[N \backslash s^{r}, s^{\prime}\right]}\left[\left[X_{j}^{r} t_{j}^{r}-f_{j}\left(X_{j}^{r}\right)\right]-\left[\left(t_{j}^{r}+\varepsilon\right) \Psi_{j}^{r}-f_{j}\left(\Psi_{j}^{r}\right)\right]\right] \\
& \sum_{\substack{j \in\left[s_{j \in\left[N \backslash N \backslash s^{r}, s^{r}\right]}[\text { or } \\
j\right.}}\left[f_{j}\left(\Psi_{j}^{r}\right)-f_{j}\left(X_{j}^{r}\right)-\left(\Psi_{j}^{r}-X_{j}^{r}\right) t_{j}^{r}\right] \\
& +\varepsilon\left[\sum_{j \in\left[s^{r}, N \backslash s^{r}\right]} \Psi_{j}^{r}-\sum_{j \in\left[N \backslash s^{r}, s^{r}\right]} \Psi_{j}^{r}\right]
\end{aligned}
$$


Since

$$
\begin{aligned}
\sum_{j \in\left[S^{\prime}, N \backslash S^{r}\right]} \Psi_{j}^{r}-\sum_{j \in\left[N \backslash S^{r}, S^{r}\right]} \Psi_{j}^{r} & =\sum_{j \in\left[S^{\prime}, N \backslash S^{r}\right]} g_{j}^{+}\left(t_{j}^{r}-\varepsilon\right)-\sum_{j \in\left[N \backslash S^{r}, S^{\prime}\right]} g_{j}^{-}\left(t_{j}^{r}+\varepsilon\right) \\
& \geqq \sum_{j \in\left[S^{\prime}, N \backslash S^{r}\right]} g_{j}^{-}\left(t_{j}^{r}-\varepsilon\right)-\sum_{j \in\left[N \backslash S^{r}, S^{r}\right]} g_{j}^{+}\left(t_{j}^{r}+\varepsilon\right) \\
& =-q^{\prime}\left(p^{r}+\varepsilon u\left(S^{r}\right) ; u\left(S^{r}\right)\right)>0
\end{aligned}
$$

(where the last strict inequality is obtained from Proposition 3.1) the left side of (32) follows. The right side of (32) follows from the convexity of $f_{j}$. QED

PROPOSITION 3.3. Under Assumption D the number of dual descent steps is finite.

Proof. We will argue by contradiction. Suppose that the number of dual descent steps is infinite. We denote the price vector, the tension vector and the flow vector generated by the relaxation algorithm at the $r$ th dual descent step by $p^{r}, t^{r}$, and $x^{r}$, respectively. First we show the following property of the sequence $\left\{t^{r}\right\}$ :

For each $j$

$$
\begin{aligned}
& \left\{t_{j}^{r}\right\}_{R} \rightarrow+\infty \text { for some subsequence } R \Rightarrow c_{j}<+\infty, \quad f_{j}\left(c_{j}\right)<\infty \\
& \left\{t_{j}^{r}\right\}_{R} \rightarrow-\infty \text { for some subsequence } R \Rightarrow l_{j}>-\infty, \quad f_{j}\left(l_{j}\right)>-\infty
\end{aligned}
$$

If $\left\{t^{r}\right\}$ is bounded then (33), (34) trivially hold. Consider a subsequence $R$ such that $\left\{t^{r}\right\}_{R}$ is unbounded. Without loss of generality suppose that, for each arc $j \in A$, $\left\{t_{j}^{r}\right\}$ is either bounded, or tends to $\infty$, or tends to $-\infty$. We now partition $N$ into a collection of nonempty subsets $N_{0}, N_{1}, \cdots, N_{L}(L \geqq 1)$ such that

$$
\left\{\left(p_{i}^{r}-p_{k}^{r}\right)\right\} \rightarrow \infty \quad \text { if } \alpha>\beta \text { and } i \in N_{\alpha}, k \in N_{\beta} \text {. }
$$

(One way to construct such a collection is to consider a graph identical to the original except that all arcs $j$ such that $\left\{t_{j}^{r}\right\}_{R}$ is bounded are discarded, and all arcs $j$ such that $\left\{t_{j}^{r}\right\}_{R} \rightarrow-\infty$ are reversed in their orientation. Since the sum of tensions along a directed cycle is zero we see that this graph is acyclic. The set $N_{0}$ is the set of nodes of this acyclic graph having no outgoing arcs. The set $N_{1}$ is obtained similarly after all arcs

For $a=1,2, \cdots, L$, we define the following arc sets:

$$
\begin{aligned}
& A_{a}^{+}=\left\{j \sim(i, k) \mid i \in \underset{\tau \geqq a}{\bigcup} N_{\tau}, k \in \underset{\tau<a}{\cup} N_{\tau}\right\}, \\
& A_{a}^{-}=\left\{j \sim(i, k) \mid i \in \underset{\tau<a}{\cup} N_{\tau}, k \in \underset{\tau \geqq a}{\cup} N_{\tau}\right\} .
\end{aligned}
$$

Then each set $A_{a}^{+} \cup A_{a}^{-}$is a cut in the network and

$$
\begin{aligned}
& t_{j}^{r} \rightarrow+\infty, r \in R \text { if and only if } j \text { belongs to some } A_{a}^{+}, \\
& t_{j}^{r} \rightarrow-\infty, r \in R \text { if and only if } j \text { belongs to some } A_{a}^{-} .
\end{aligned}
$$

Consider any fixed positive scalar $\Delta$. Equation (35) implies that for all $a$

$$
\begin{gathered}
\lim _{r \rightarrow \infty, r \in R} g_{j}^{-}\left(t_{j}^{r}-\Delta\right)=c_{j} \quad \forall j \in A_{a}^{+}, \quad \lim _{r \rightarrow \infty, r \in R} g_{j}^{+}\left(t_{j}^{r}+\Delta\right)=l_{j} \quad \forall j \in A_{a}^{-} . \\
q^{\prime}\left(p^{r}+\Delta u ; u\right)=-\sum_{j \in A_{a}^{+}} g_{j}^{-}\left(t_{j}^{r}-\Delta\right)+\sum_{j \in A_{a}^{-}} g_{j}^{+}\left(t_{j}^{r}+\Delta\right)
\end{gathered}
$$


where $u$ is given by

$$
u_{i}= \begin{cases} & \text { if } i \in \underset{r \geq a}{\bigcup} N_{\tau}, \\ 0 & \text { otherwise, }\end{cases}
$$

it follows from (36) that

$$
\lim _{r \rightarrow \infty, r \in R} q^{\prime}\left(p^{r}+\Delta u ; u\right)=-\sum_{j \in A_{a}^{+}} c_{j}+\sum_{j \in A_{a}^{-}} l_{j} .
$$

Let $\Theta$ denote the right-hand side quantity in (37). We will argue that $\Theta=0$. Clearly we cannot have $\Theta>0$ since this would imply that there does not exist a primal feasible solution. We also cannot have $\Theta<0$ since then (37) implies that for $r$ sufficiently large

$$
q\left(p^{r}+\Delta u\right) \leqq q\left(p^{r}\right)+\Delta \Theta .
$$

This is not possible since $\Delta$ can be chosen arbitrarily large while $q\left(p^{r}\right)$ is nonincreasing with $r$. This leaves the only possibility that $\Theta=0$ or that

$$
\sum_{j \in A_{a}^{+}} c_{j}=\sum_{j \in A_{a}^{-}} l_{j}, \quad a=1, \cdots, L .
$$

It follows that for every feasible flow vector we have

$$
x_{j}=c_{j} \quad \forall j \in A_{a}^{+}, \quad x_{j}=l_{j} \quad \forall j \in A_{a}^{-}, \quad a=1, \cdots, L .
$$

This implies (33) and (34).

Now we will bound from below the amount of improvement in the dual cost per dual descent step by a positive constant. Proposition 3.1 assures us that at each dual descent step the step length is more than $\varepsilon$. Consider the interval $\left[\frac{1}{4} \varepsilon, \frac{3}{4} \varepsilon\right]$, which we denote by $I$. Also let $u^{r}$ denote the dual descent direction at the $r$ th dual descent step. We have that the dual cost is decreasing on the line segment connecting $t^{r}$ and $t^{r+1}$. It follows from (33), (34) and Assumption $D$ that there exists a subsequence $R$ such that for $r$ sufficiently large, $r \in R$, we have for all $\Delta \in I$

$$
\begin{aligned}
q^{\prime}\left(p^{r}+\Delta u^{r} ; u^{r}\right)= & \sum_{j \in J^{+}} c_{j} v_{j}^{r}+\sum_{j \in J^{-}} l_{j} v_{j}^{r}+\sum_{\substack{j \in J^{0} \\
v j>0}} g_{j}^{+}\left(t_{j}^{r}+\Delta v_{j}^{r}\right) v_{j}^{r} \\
& +\sum_{\substack{j \in J^{0} \\
v j<0}} g_{j}^{-}\left(t_{j}^{r}+\Delta v_{j}^{r}\right) v_{j}^{r}<0
\end{aligned}
$$

where we define

$$
\begin{aligned}
& J^{+}=\left\{j \mid\left\{t_{j}^{r}\right\}_{r \in R} \rightarrow \infty\right\}, \quad J^{-}=\left\{j \mid\left\{t_{j}^{r}\right\}_{r \in R} \rightarrow-\infty\right\}, \\
& J^{0}=\left\{j \mid\left\{t_{j}^{r}\right\}_{r \in R} \text { is bounded }\right\}
\end{aligned}
$$

and $v^{r}=E^{T} u^{r}$. Consider a fixed $r \in R$. Define $\Theta: R \rightarrow R$ by

$$
\Theta(\Delta)=q\left(p^{r}+\Delta u^{r}\right)
$$

We consider two cases. In case (i) the right derivative of $\Theta(\Delta)$ assumes at most $2|A|$ distinct values in the interval $I$. In case (ii) the right derivative of $\Theta(\Delta)$ assumes more than $2|A|$ distinct values in the interval $I$. In case (i) $q\left(p^{r}+\Delta u^{r}\right)$ is linear for $\Delta$ in some subinterval $I^{r}$ of $I$ of length at least $\varepsilon / 4|A|$ and it follows that $q^{\prime}\left(p^{r}+\Delta u^{r} ; u^{r}\right)$ over $I^{r}$ is linear of the form

$$
q^{\prime}\left(p^{r}+\Delta u^{r} ; u^{r}\right)=\sum_{j \in J^{+}} c_{j} v_{j}^{r}+\sum_{j \in J^{-}} l_{j} v_{j}^{r}+\sum_{j \in J^{0}} b_{j} v_{j}^{r}
$$


where $v^{r}=E^{T} u^{r}$ and $b_{j}$ denotes some breakpoint of $f_{j}$. This implies that, for each $j \in J^{0}$ such that $v_{j}^{r} \neq 0$, the dual functional $g_{j}\left(t_{j}^{r}+\Delta v_{j}^{r}\right)$ is linear with slope $b_{j}$ for $\Delta$ in $I^{r}$. For each $j \in J^{0},\left\{t_{j}^{r}\right\}_{r \in R}$ is bounded, and therefore the number of distinct linear pieces of $g_{j}$ of length $\geqq \varepsilon / 4|A|$ encountered during the course of the algorithm is finite. This together with the fact that $v^{r}$ is chosen from a finite set imply that $q^{\prime}\left(p^{r}+\Delta u^{r} ; u^{r}\right)$ (cf. (38)) can only assume one of a finite set of values over the subinterval $\boldsymbol{I}^{r}$. It follows that in case (i) we can bound the amount of dual cost improvement from below by $\delta \varepsilon / 4|A|$ where $\delta$ is some positive scalar. This implies that case (i) can occur for only a finite set of indexes $r$ (for otherwise the dual cost tends to $-\infty$ ) and we need only to consider case (ii). In case (ii) for each $r \in R$ there must exist a $j \in J^{0}$ such that $v_{j}^{r} \neq 0$ and the right derivative of the function $h(\Delta)$ defined by $h(\Delta)=g_{j}\left(t_{j}^{r}+\Delta v_{j}^{r}\right)$ assumes at least three distinct values in the interval $I$. Since $v_{j}^{r}$ equals either 1 or -1 it follows that either $t_{j}^{r+1} \geqq t_{j}^{r}+\varepsilon$ and $g_{j}^{+}\left(t_{j}^{r}+\Delta_{1}\right)<g_{j}^{-}\left(t_{j}^{r}+\Delta_{2}\right)$ for at least two points $\Delta_{1}<\Delta_{2}$ in $I$ or $t_{j}^{r+1} \leqq t_{j}^{r}-\varepsilon$ and $g_{j}^{+}\left(t_{j}^{r}-\Delta_{2}\right)<g_{j}^{-}\left(t_{j}^{r}-\Delta_{1}\right)$ for least two points $\Delta_{1}<\Delta_{2}$ in $I$. Passing to a subsequence if necessary, we can assume that it is the same $j$ and either $t_{j}^{r+1} \geqq t_{j}^{r}+\varepsilon$ or $t_{j}^{r+1} \leqq t_{j}^{r}-\varepsilon$ for all $r \in R$ that are sufficiently large. Without loss of generality we will assume that $t_{j}^{r+1} \geqq t_{j}^{r}+\varepsilon$ for all $r \in R$ that are sufficiently large. Since $j \in J^{0}$ the subsequence $\left\{t_{j}^{\prime}\right\}_{r \in R}$ is bounded and therefore has a limit point $t_{j}^{*}$. Passing to a subsequence if necessary, we assume that $\left\{t_{j}^{r}\right\}$ converges to $t_{j}^{*}$. Then it follows that there exists a fixed interval $L$ such that

$$
\begin{gathered}
L \subset\left[t_{j}^{r}, t_{j}^{r}+\varepsilon\right] \quad \forall r \in R, r \text { sufficiently large } \\
\eta_{1}<\eta_{2} \text { and } g_{j}^{+}\left(\eta_{1}\right)<g_{j}^{-}\left(\eta_{2}\right)
\end{gathered}
$$

for at least two distinct points $\eta_{1}$ and $\eta_{2}$ in $L$. We then define

$$
\xi_{1}=g_{j}^{-}\left(\eta_{1}\right), \quad \xi_{2}=g_{j}^{+}\left(\eta_{2}\right)
$$

Then $\xi_{1}$ and $\xi_{2}$ belong to the interval

$$
\left[g_{j}^{-}(a), g_{j}^{+}(b)\right]
$$

where $a, b$ are the left and the right endpoints of $L$, respectively, and they satisfy

$$
\xi_{1}<\xi_{2} \text { and } f_{j}^{+}\left(\xi_{1}\right)<f_{j}^{-}\left(\xi_{2}\right) \text {. }
$$

Then for $r$ sufficiently large, $r \in R$, we obtain (cf. (39)) that

$$
g_{j}^{+}\left(t_{j}^{r}\right) \leqq \xi_{1}<\xi_{2} \leqq g_{j}^{-}\left(t_{j}^{r}+\varepsilon\right) .
$$

It follows from Proposition 3.2 that for all sufficiently large $r \in \boldsymbol{R}$

$$
\begin{aligned}
q\left(p^{r}\right)-q\left(p^{r+1}\right) & \geqq f_{j}\left(g_{j}^{-}\left(t_{j}^{r}+\varepsilon\right)\right)-f_{j}\left(g_{j}^{+}\left(t_{j}^{r}\right)\right)-f_{j}^{+}\left(g_{j}^{+}\left(t_{j}^{r}\right)\right)\left(g_{j}^{-}\left(t_{j}^{r}+\varepsilon\right)-g_{j}^{+}\left(t_{j}^{r}\right)\right) \\
& \geqq f_{j}\left(\xi_{2}\right)-f_{j}\left(\xi_{1}\right)-f_{j}^{+}\left(\xi_{1}\right)\left(\xi_{2}-\xi_{1}\right)
\end{aligned}
$$

where the second inequality follows from (41) and the convexity of $f_{j}$. From (40) and the convexity of $f_{j}$ we obtain that the right-hand side of $(42)$ is positive. Therefore the dual cost improvement per dual descent is bounded from below by a positive constant, and the dual cost tends to $-\infty$, contradicting Assumption A. QED

The second part of our finite termination proof involves showing that the number of flow augmentations between successive dual descent steps is finite. Since the $\varepsilon$-bounds remain unchanged between successive dual descent steps, the issue in effect is whether the labeling algorithm used will solve finitely the max flow problem with the given $\varepsilon$-bounds taken as capacity constraints. It was shown by Ford and Fulkerson 
([14, p. 126]) that, when the data is irrational, an arbitrary choice of labeled nodes may result in an infinite number of flow augmentations, so a more specific scheme for labeling is necessary to deal with irrational data. Here we propose two such schemes: breadth-first search and arc discrimination. In practice, the data is always rational, being stored on a finite precision machine, and therefore finite convergence is assured even if labeling is done arbitrarily.

Breadth-first search is a well-known scheme used in labeling. It can be easily implemented using a FIFO queue. In $[15$, Chap. 9.3, 16] it was shown that, under breadth-first search, the number of flow augmentations is finite if all nodes with positive deficit are labeled initially. We now show that the same conclusion holds if a single node with positive deficit is labeled initially, as is the case for the relaxation iteration. This fact requires a nontrivial proof and, to our knowledge, is not reported in the literature.

PROPOSITION 3.4. When labeling is done by breadth-first search, the number of flow augmentations between successive dual descent steps is finite.

Proof. We will assume that the number of flow augmentations is infinite and obtain a contradiction. For simplicity, we call a node with negative deficit a source and a node with positive deficit a sink. Since the number of flow augmentations is infinite, after a while the set of sources and sinks must become fixed (since a source cannot become a sink or vice versa) and the set of flow augmenting paths must repeat (since all flow augmenting paths are simple and therefore there are only a finite number of them). Let $P$ be the set of flow augmenting paths that repeat infinitely often. We say that an arc belonging to a path $p \in P$ is saturated in the direction of $p$ if the flow of the arc is at the upper (lower) bound and the arc is oriented from the source (sink) of $p$ to the sink (source) of $p$.

Consider a path $p \in P$. After a flow augmentation using $p$ as the path, some arc of $p$ will become saturated in the direction of $p$. Let $A_{p}$ denote the set of arcs on $p$ that become saturated in the direction of $p$ infinitely often. $A_{p}$ is clearly nonempty. We will show, by induction, that $A_{p}$ is empty when breadth-first search is done and thus obtain a contradiction.

Initialization. For all $p \in P$, every $a \in A_{p}$ is at least one arc away from the sink $t$ of $p$.

Proof. This is true since if the arc on $p$ incident to $t$ is saturated, it must remain saturated from then on.

$k t h$ inductive step. Suppose that, for all $p \in P$, every $a \in A_{p}$ is at least $k$ arcs away from the sink of $p$. We will show that, for all $p \in P$, every $a \in A_{p}$, is $k+1$ arcs away from the sink of $p$. Suppose the contrary. Then there exists a $p \in P$, whose source and sink we denote by $s$ and $t$ respectively, and an arc $a$ in $A_{p}$ such that $a$ is $k$ arcs away from $t$. After $a$ becomes saturated, there must be a flow augmenting path $p^{\prime}$ to unsaturate it (see Fig. 3.4). From the inductive hypothesis, the arcs on $p$ between $a$ and $t$ are unsaturated in the direction of $p$. Since the labeling is done by breadth-first search,

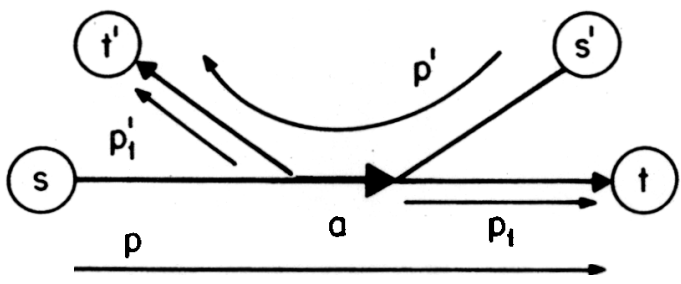

FiG. 3.4. p' unsaturating the arc a. 
this implies that the number of arcs on the subpath $p_{1}^{\prime}$ (see Fig. 3.4) must be strictly less than that of the subpath $p_{1}$ (otherwise during the iteration that generated $p^{\prime}$ as the flow augmenting path node $t$ would have been labeled before node $\left.t^{\prime}\right)$. It follows that, just before the iteration that generated path $p$, some arc of the subpath $p_{1}^{\prime}$ must be saturated in the direction of $p^{\prime}$ (otherwise during the iteration that generated $p$ node $t^{\prime}$ would be labeled before node $t$ ). This arc must then belong to $A_{p^{\prime}}$. Since the number of arcs on $p_{1}^{\prime}$ is strictly less than $k$, the inductive hypothesis is contradicted.

Since the inductive hypothesis holds for all $k$ and the number of arcs on each flow augmenting path is at most $|N|-1$, it follows that $A_{p}$ is empty for all $p$ and the desired contradiction is obtained. QED

In the arc discrimination scheme, the order in which nodes are labeled and scanned is given by the following simple rule:

Each labeled but unscanned node records whether it is connected to an unlabeled neighbor by an arc whose flow is strictly between the lower and upper bounds.

A node with such a neighbor is scanned first.

The proof of finite convergence under this scheme is given in [17]. The implementation of the arc discrimination scheme requires more global information than breadthfirst search. However, when the relaxation algorithm is extended to operate on both positive and negative deficit nodes between successive dual descent steps, which had been shown to be computationaly beneficial in the case of linear cost problems, arc discrimination can still be shown to yield finite convergence. It is not known if this is also true of breadth-first search.

Propositions 3.3 and 3.4 show that the relaxation algorithm of this section terminates after a finite number of iterations. Since the algorithm only terminates when all the node deficits have zero value, the final flow vector $x$ must belong to $C$. Since $\varepsilon$-CS is maintained at all iterations of the algorithm, it follows that $x$ and the final dual price vector must satisfy $\varepsilon$-CS also.

We next show that we can bring the cost of the solution generated by the relaxation algorithm arbitrarily close to the optimal cost by taking $\varepsilon$ sufficiently small. The main part of the argument is embodied in the next proposition.

Proposition 3.5. Let $x$ and $p$ satisfy $\varepsilon$-CS, and let $\xi$ and $p$ satisfy CS. If $x \in C$ then

$$
0 \leqq f(x)+q(p) \leqq \varepsilon \sum_{j \in A}\left|x_{j}-\xi_{j}\right| \text {. }
$$

Proof. Let $t=E^{T} p$. Since $\xi$ and $p$ satisfy CS we have

$$
f_{j}\left(\xi_{j}\right)=\xi_{j} t_{j}-g_{j}\left(t_{j}\right) \quad \forall j \in A .
$$

Take an arc $j$ such that $x_{j} \geqq \xi_{j}$. Then by convexity of $f_{j}$

$$
f_{j}\left(x_{j}\right)+\left(\xi_{j}-x_{j}\right) f_{j}^{-}\left(x_{j}\right) \leqq f_{j}\left(\xi_{j}\right)=\xi_{j} t_{j}-g_{j}\left(t_{j}\right) .
$$

Hence

$$
\begin{aligned}
f_{j}\left(x_{j}\right)+g_{j}\left(t_{j}\right) & \leqq\left(x_{j}-\xi_{j}\right)\left(f_{j}^{-}\left(x_{j}\right)-t_{j}\right)+x_{j} t_{j} \\
& \leqq\left|x_{j}-\xi_{j}\right| \varepsilon+x_{j} t_{j}
\end{aligned}
$$

where the second inequality follows from $\varepsilon$-CS. This inequality is similarly obtained when $x_{j} \leqq \xi_{j}$, so we have

$$
f_{j}\left(x_{j}\right)+g_{j}\left(t_{j}\right) \leqq\left|x_{j}-\xi_{j}\right| \varepsilon+x_{j} t_{j} \quad \forall j \in A
$$

From the definition of $g_{j}$ we also have

$$
x_{j} t_{j} \leqq f_{j}\left(x_{j}\right)+g_{j}\left(t_{j}\right) \quad \forall j \in A .
$$


By combining these two inequalities and adding over all $j \in A$, we obtain

$$
\sum_{j \in A} x_{j} t_{j} \leqq \sum_{j \in A}\left[f_{j}\left(x_{j}\right)+g_{j}\left(t_{j}\right)\right] \leqq \varepsilon \sum_{j \in A}\left|x_{j}-\xi_{j}\right|+\sum_{j \in A} x_{j} t_{j} .
$$

Since $x \in C$ we have $\sum_{j \in A} x_{j} t_{j}=0$ and the result follows. QED

From Proposition 3.5 we can obtain a simple bound on the suboptimality of the solution in the special case where $l_{j}>-\infty$ and $c_{j}<+\infty$ for all $j \in A$.

CoROllary 3.1. Let $x$ and p satisfy $\varepsilon$-CS. If $x \in C$, and $-\infty<l_{j} \leqq c_{j}<+\infty$ for all $j \in A$, then

$$
0 \leqq f(x)+q(p) \leqq \varepsilon \sum_{j \in A}\left(c_{j}-l_{j}\right)
$$

For the general case we have the following.

PROPOSITION 3.6. Let $x(\varepsilon)$ and $p(\varepsilon)$ denote any flow and price vector pair such that $x(\varepsilon)$ and $p(\varepsilon)$ satisfy $\varepsilon$-CS and $x(\varepsilon) \in C$. Then $f(x(\varepsilon))+q(p(\varepsilon)) \rightarrow 0$ as $\varepsilon \rightarrow 0$.

Proof. First we show that $x(\varepsilon)$ remains bounded as $\varepsilon \rightarrow 0$. If $x(\varepsilon)$ is not bounded as $\varepsilon \rightarrow 0$, then since $x(\varepsilon) \in C$ for all $\varepsilon>0$ there exists a directed cycle $Y$ and a sequence $\left\{\varepsilon_{n}\right\} \rightarrow 0$ such that $c_{j}=+\infty, x_{j}\left(\varepsilon_{n}\right) \rightarrow+\infty$ for all $j \in Y^{+}$and $l_{j}=-\infty, x_{j}\left(\varepsilon_{n}\right) \rightarrow-\infty$ for all $j \in Y^{-}$. By Assumption B

$$
\lim _{\xi \rightarrow+\infty} f_{j}^{-}(\xi)=+\infty \quad \text { for all } j \in Y^{+}, \quad \lim _{\xi \rightarrow-\infty} f_{j}^{+}(\xi)=-\infty \text { for all } j \in Y^{-} .
$$

This implies that for $\boldsymbol{n}$ sufficiently large,

$$
t_{j}\left(\varepsilon_{n}\right)>t_{j}\left(\varepsilon_{0}\right) \text { for all } j \in Y^{+} \text {and } t_{j}\left(\varepsilon_{n}\right)<t_{j}\left(\varepsilon_{0}\right) \text { for all } j \in Y^{-}
$$

where $t\left(\varepsilon_{n}\right)=E^{T} p\left(\varepsilon_{n}\right)$. Since $t\left(\varepsilon_{n}\right)=E^{T} p\left(\varepsilon_{n}\right)$ we have

$$
\sum_{j \in Y^{+}} t_{j}\left(\varepsilon_{n}\right)-\sum_{j \in Y^{-}} t_{j}\left(\varepsilon_{n}\right)=0 \text { for all } n
$$

which contradicts (43). Therefore $x(\varepsilon)$ is bounded as $\varepsilon \rightarrow 0$.

Now we will show that $\xi_{j}(\varepsilon)-x_{j}(\varepsilon)$ is bounded for all $j \in A$ as $\varepsilon \rightarrow 0$, where $\xi(\varepsilon)$ is some vector satisfying $f_{j}^{-}\left(\xi_{j}(\varepsilon)\right) \leqq t_{j}(\varepsilon) \leqq f_{j}^{+}\left(\xi_{j}(\varepsilon)\right.$ ), for all $j \in A$. If $c_{j}<\infty$ then $\xi_{j}(\varepsilon)$ is trivially bounded from above. If $c_{j}=+\infty$ then by Assumption B we have $f_{j}^{-}(\xi) \rightarrow+\infty$ as $\xi \rightarrow+\infty$. Since $x_{j}(\varepsilon)$ is bounded we have that $t_{j}(\varepsilon)$ is bounded from above which in turn implies that $\xi_{j}(\varepsilon)$ is bounded from above. Similarly, we can argue that $\xi_{j}(\varepsilon)$ is bounded from below. Therefore $\left|\xi_{j}(\varepsilon)-x_{j}(\varepsilon)\right|$ is bounded for all $j \in A$ as $\varepsilon \rightarrow 0$. This then completes our proof in view of Proposition 3.5. QED

Unfortunately Proposition 3.6 does not tell us how small $\varepsilon$ must be to achieve a certain degree of near optimality. We need to solve the problem first for some guess $\varepsilon$ to obtain $x(\varepsilon)$ and $\xi(\varepsilon)$, evaluate the quality of the solution on the basis of the gap $f(x(\varepsilon))+q(p(\varepsilon))$ between primal and dual solution, and then decide whether $\varepsilon$ needs to be decreased. If however the bounds $l_{j}$ and $c_{j}$ are finite, we can, by Corollary 3.1, obtain an a priori estimate on $\varepsilon$.

4. Computational experimentation. Two experimental codes implementing the methods of the paper were developed and tested on linear benchmark problems and nonlinear variations.

The first code, named NRELAX, implements the relaxation method for strictly convex problems of $\S 2$. The second code, named MNRELAX, implements the method for mixed linear and strictly convex problems of $\S 3$. Both codes were written in Fortran on a VAX 11-750 and were compiled and run under the VMS version 3.7 operating system. 
TABLE 1

Times for NETGEN benchmark problems. MNRELAX uses $\varepsilon=0$. Times in this and subsequent tables are in secs on a VAX 11-750. All codes are written in Fortran and compiled under VMS version 3.7.

\begin{tabular}{|c|c|c|c|c|}
\hline $\begin{array}{l}\text { Problem } \\
\text { number }\end{array}$ & $\begin{array}{c}\text { Number of } \\
\text { nodes }\end{array}$ & $\begin{array}{c}\text { Number of } \\
\text { arcs }\end{array}$ & $\begin{array}{c}\text { MNRELAX } \\
\varepsilon=0\end{array}$ & RELAX-II \\
\hline & 200 & 1300 & 5.13 & 2.07 \\
\hline & 200 & 1500 & 6.33 & 2.12 \\
\hline & 200 & 2000 & 4.86 & 1.92 \\
\hline & 200 & 2200 & 7.74 & 2.52 \\
\hline & 200 & 2900 & 6.83 & 2.97 \\
\hline & 300 & 3150 & 12.85 & 4.37 \\
\hline 7 & 300 & 4500 & 13.46 & 5.46 \\
\hline 8 & 300 & 5155 & 14.54 & 5.39 \\
\hline 9 & 300 & 6075 & 17.38 & 6.38 \\
\hline \multirow[t]{2}{*}{10} & 300 & 6300 & 14.39 & 4.12 \\
\hline & 400 & 1500 & 4.71 & 1.23 \\
\hline 12 & 400 & 2250 & 5.81 & 1.38 \\
\hline 13 & 400 & 3000 & 6.27 & 1.68 \\
\hline 14 & 400 & 3750 & 7.79 & 2.43 \\
\hline 15 & 400 & 4500 & 9.64 & 2.79 \\
\hline 16 & 400 & 1306 & 9.06 & 2.79 \\
\hline 17 & 400 & 2443 & 8.87 & 2.67 \\
\hline 18 & 400 & 1306 & 8.98 & 2.56 \\
\hline 19 & 400 & 2443 & 8.81 & 2.73 \\
\hline 20 & 400 & 1416 & 9.82 & 2.85 \\
\hline 21 & 400 & 2836 & 10.36 & 3.80 \\
\hline 22 & 400 & 1416 & 9.08 & 2.56 \\
\hline 23 & 400 & 2836 & 13.80 & 4.91 \\
\hline 24 & 400 & 1382 & 4.73 & 1.27 \\
\hline 25 & 400 & 2676 & 7.15 & 2.01 \\
\hline 26 & 400 & 1382 & 3.73 & 1.79 \\
\hline 27 & 400 & 2676 & 6.41 & 2.15 \\
\hline 28 & 1000 & 2900 & 20.17 & 4.90 \\
\hline 29 & 1000 & 3400 & 19.15 & 5.57 \\
\hline 30 & 1000 & 4400 & 25.62 & 7.31 \\
\hline
\end{tabular}

The test problems were generated using the public domain code NETGEN [18]. There are 40 "standard" benchmark linear cost problems that can be obtained using this code. We tested our codes with some of these problems either in their standard (linear cost) form or in a modified form whereby a quadratic cost was added to the linear cost of some or all of the arcs as discussed below. In order to test coding efficiency we tested MNRELAX with $\varepsilon=0$ against the very efficient linear cost code RELAX-II (see [1], [2]) under identical conditions on the first 30 NETGEN benchmark problems. The two codes are close to being mathematically equivalent on linear cost problems but MNRELAX uses floating point arithmetic. The results shown in Table 1 appear to indicate that MNRELAX is coded fairly efficiently.

There were two issues that we wanted to clarify through the experimentation:

(a) The effect of the parameter $\varepsilon$ on the performance of MNRELAX;

(b) The relative efficiency of NRELAX versus MNRELAX with optimal choice of the parameter $\varepsilon$ on strictly convex problems.

A large number of experiments some of which are presented in Tables 2 and 3 showed that for all except some very "difficult" problems it is best to operate 
TABLE 2

Times for NETGEN benchmark problems modified so that $50 \%$ of the ares hate an additional quadratic cost with coefficient from the range $[5,10]$. Numbers in parentheses where present indicate significant digits of accuracy of the answer. In MNRELAX $\varepsilon$ is kept constant during the solution of each problem.

\begin{tabular}{|c|c|c|c|c|c|}
\hline $\begin{array}{l}\text { Problem } \\
\text { number }\end{array}$ & $\begin{array}{l}\text { Number of } \\
\text { nodes }\end{array}$ & $\begin{array}{c}\text { Number of } \\
\text { ares }\end{array}$ & $\begin{array}{c}\text { MNRELAX } \\
x>0\end{array}$ & $\begin{array}{c}\text { MNRELAX } \\
E=0\end{array}$ & $\begin{array}{c}\text { Sign, digits of } \\
\text { accuracy }\end{array}$ \\
\hline 1 & 200 & 1300 & 30.79 & 11.49 & 6 \\
\hline 2 & 200 & 1500 & 40.66 & 11.73 & 6 \\
\hline 3 & 200 & 2000 & 34,48 & 9.31 & 7 \\
\hline 4 & 200 & 2200 & 32.05 & 11.60 & 6 \\
\hline 5 & 200 & 2900 & 50.43 & 28.14 & 5 \\
\hline 6 & 300 & 3150 & 74.10 & 26.01 & 6 \\
\hline 7 & 300 & 4500 & 140,70 & 48.64 & 6 \\
\hline 8 & 300 & siss & 116.06 & 76.35 & 6 \\
\hline 9 & 300 & 6075 & 96.59 & 49.25 & 6 \\
\hline 10 & 300 & 6300 & 94.71 & 36.43 & 6 \\
\hline 11 & 400 & 1500 & 263.14 & 26.35 & 4 \\
\hline 12 & 400 & 2250 & 180.93 & 31.86 & 4 \\
\hline 13 & 400 & 3000 & 240.76 & (5) 36.25 & 4 \\
\hline 14 & 400 & 3750 & 436.80 & 79.88 & 4 \\
\hline 15 & 400 & 4500 & 146.69 & (3) 42.23 & 2 \\
\hline 16 & 400 & 1306 & 144.08 & (7) 86.40 & 3 \\
\hline 17 & 400 & 2443 & 261.91 & (7) 47.31 & 5 \\
\hline 18 & 400 & 1306 & 294.88 & 53.71 & 4 \\
\hline 19 & 400 & 2443 & 108.04 & 37.54 & 5 \\
\hline 20 & 400 & 1416 & 21499 & (7) 68.17 & 3 \\
\hline 21 & 400 & 2836 & 37.24 & (7) 18.43 & 4 \\
\hline 22 & 400 & 1416 & 366.85 & (7) 53.37 & 4 \\
\hline 23 & 400 & 2836 & 34.56 & (7) 18.09 & 4 \\
\hline 24 & 400 & 1382 & 66.58 & (5) 45.87 & 3 \\
\hline$n$ & 400 & 2676 & 167.53 & (6) 22.73 & 5 \\
\hline
\end{tabular}

MNRELAX with $\varepsilon=0$ and terminate the iterations when the deficit of all nodes becomes sufficiently close to zero. Indeed it appears that for such problems the time required for MNRELAX to terminate increases with $\varepsilon$. The reason is probably that with large $\varepsilon$ the intervals defined by the $\varepsilon$-bounds become larger and, as a result, a large number of flow augmentations are needed before a descent direction can be found. Given that a large value of $\varepsilon$ leads also to inaccurate solutions (see Proposition 3.5), it appears that for most problems the best way to operate MNRELAX is with $\varepsilon=0$ or with $\varepsilon$ very small.

When $\varepsilon=0$ and all arc costs are strictly convex, MNRELAX and NRELAX are mathematically equivalent. However NRELAX is somewhat faster because of more efficient coding as shown in Table 3.

Finally in Table 4 we show results obtained on some "difficult" problems with strictly convex arc costs. These problems were constructed by choosing the quadratic cost efficients of some arcs to be very small relative to others as described in Table 4. This is similar to a situation in nonlinear unconstrained minimization where the Hessian matrix of the cost function has some eigenvalues that are very small relative to other eigenvalues. For this class of problems MNRELAX with nonzero $\varepsilon$ can outperform both NRELAX and MNRELAX with $\varepsilon=0$. This is not surprising in view of the coordinate descent interpretation of NRELAX. The version of MNRELAX that we found most efficient for these problems is one whereby we start with a moderate value 
TABLE 3

Times for NETGEN benchmark problems modified so that all arcs have an additional quadratic cost with coefficient from the range $[5,10]$. Numbers in parentheses where present indicate significant digits of accuracy of the answer. In MNRELAX $\varepsilon$ is kept constant during solution of each problem.

\begin{tabular}{|c|c|c|c|c|c|c|}
\hline $\begin{array}{l}\text { Problem } \\
\text { number }\end{array}$ & $\begin{array}{c}\text { Number of } \\
\text { nodes }\end{array}$ & $\begin{array}{c}\text { Number of } \\
\text { arcs }\end{array}$ & $\begin{array}{c}\text { MNRELAX } \\
\varepsilon>0\end{array}$ & $\begin{array}{c}\text { MNRELAX } \\
\varepsilon=0\end{array}$ & NRELAX & $\begin{array}{l}\text { Sign. digits of } \\
\text { accuracy }\end{array}$ \\
\hline & 200 & 1300 & 22.63 & 17.97 & 10.80 & 7 \\
\hline 2 & 200 & 1500 & 23.37 & 19.12 & 11.51 & 7 \\
\hline 3 & 200 & 2000 & 19.33 & 20.77 & 12.04 & 8 \\
\hline 4 & 200 & 2200 & 37.87 & 25.38 & 17.22 & 7 \\
\hline 5 & 200 & 2900 & 34.82 & 32.37 & 21.44 & 6 \\
\hline 6 & 300 & 3150 & 113.75 & 57.95 & 40.23 & 7 \\
\hline 7 & 300 & 4500 & 85.26 & 50.49 & 37.77 & 6 \\
\hline 8 & 300 & 5155 & 95.11 & 70.08 & 49.38 & 8 \\
\hline 9 & 300 & 6075 & 70.48 & 69.44 & 48.04 & 7 \\
\hline 10 & 300 & 6300 & (6)99.69 & 69.33 & 41.41 & 5 \\
\hline 11 & 400 & 1500 & (7) 43.19 & 34.37 & 14.67 & 6 \\
\hline 12 & 400 & 2250 & (6)39.56 & 33.31 & 12.98 & 5 \\
\hline 13 & 400 & 3000 & (7) 34.62 & 32.66 & 18.34 & 5 \\
\hline 14 & 400 & 3750 & (6) 34.97 & 35.32 & 20.86 & 5 \\
\hline 15 & 400 & 4500 & 64.90 & 42.53 & 24.95 & 5 \\
\hline 16 & 400 & 1306 & 65.86 & 54.19 & 21.54 & 6 \\
\hline 17 & 400 & 2443 & 60.62 & 46.20 & 21.89 & 6 \\
\hline 18 & 400 & 1306 & 84.26 & 72.41 & 48.97 & 7 \\
\hline 19 & 400 & 2443 & 60.56 & 46.18 & 20.80 & 6 \\
\hline 20 & 400 & 1416 & 108.49 & 72.11 & 38.70 & 7 \\
\hline 21 & 400 & 2836 & 62.78 & 38.79 & 38.69 & \\
\hline 22 & 400 & 1416 & 95.91 & 55.25 & 42.03 & \\
\hline \multirow[t]{2}{*}{23} & 400 & 2836 & 43.41 & 33.21 & 20.70 & \\
\hline & 400 & 1382 & 59.83 & 65.35 & 42.47 & \\
\hline 25 & 400 & 2676 & 53.57 & 42.06 & 37.88 & \\
\hline
\end{tabular}

of $\varepsilon$, operate MNRELAX to termination, then reduce $\varepsilon$ by a factor of 10 and repeat the process up to the point where primal and dual values differ by a specified accuracy. Still, the proper starting value for $\varepsilon$ was not easy to determine and it was necessary to do some initial experimentation with several of these difficult problems. The conclusion is that the methods of this paper may not be successful for such problems. We do not know, however, of a better alternative.

TABLE 4

Times for NETGEN benchmark problems modified so that all arcs have an additional quadratic term. In $50 \%$ of the arcs the quadratic cost coefficient was small as indicated. In the other $50 \%$ of the arcs the quadratic cost coefficient was from the range [5, 10]. Numbers in parentheses where present indicate significant digits of accuracy of the answer. In MNRELAX $\varepsilon$ is progressively decreased during solution of each problem.

\begin{tabular}{|c|c|c|c|c|c|c|}
\hline \multirow[t]{2}{*}{$\begin{array}{l}\text { Problem } \\
\text { number }\end{array}$} & & & \multirow{2}{*}{$\begin{array}{c}\begin{array}{c}\text { Small quad } \\
\text { coeff. }\end{array} \\
.0001\end{array}$} & \multicolumn{2}{|c|}{$\begin{array}{c}\text { MNRELAX } \\
\varepsilon>0\end{array}$} & \multirow{2}{*}{$\frac{\text { NRELAX }}{\text { (3) } 58.00}$} \\
\hline & 200 & 1300 & & (4) 52.98 & & \\
\hline & 200 & 2900 & .001 & (5) 227.93 & (2)27.16 & (2) 50.15 \\
\hline & 300 & 4500 & .0001 & (3) 301.03 & (2) 131.10 & (2) 218.24 \\
\hline & 400 & 1500 & .0001 & (4) 111.36 & & - \\
\hline 15 & 400 & 4500 & .01 & (3)361.71 & & (3) 1957.70 \\
\hline 16 & 400 & 1306 & .001 & (3) 287.13 & & (2) 350.97 \\
\hline 18 & 400 & 1306 & .001 & (3) 188.78 & & (2) 321.76 \\
\hline 24 & 400 & 1382 & .001 & (4) 417.83 & (2) 46.21 & (2) 134.51 \\
\hline
\end{tabular}




\section{REFERENCES}

[1] D. P. BERTSEKAS, A unified framework for primal-dual methods in minimum cost network flow problems, Math. Programming, 32 (1985), pp. 125-145.

[2] D. P. BERTSEKAS AND P. TSENG, Relaxation methods for minimum cost ordinary and generalized network flow problems, LIDS Report P-1462, Massachusetts Inst. of Technology, May 1985.

[3] R. T. Rockafellar, Network Flows and Monotropic Programming, Wiley-Interscience, New York, 1983.

[4] —, Convex Analysis, Princeton Univ. Press, Princeton, NJ, 1970.

[5] D. P. BERTSEKAS AND D. El BAZ, Distributed asynchronous relaxation methods for convex network flow problems, LIDS Report P-1417, Massachusetts Inst. of Technology, October 1984, this Journal, 25 (1987), pp. 74-85.

[6] W. I. ZANGwill, Nonlinear Programming, Prentice-Hall, Englewood Cliffs, NJ, 1969.

[7] R. W. H. SARGENT AND D. J. SEBASTIAN, On the convergence of sequential minimization algorithms, J. Optim. Theory Appl., 12 (1973), pp. 567-575.

[8] E. PolaK, Computational Methods in Optimization: A Unified Approach, Academic Press, New York, 1971.

[9] D. G. Luenberger, Linear and Nonlinear Programming, Addison-Wesley, Reading, MA, 1984.

[10] M. J. D. PowELL, On search directions for minimization algorithms, Math. Programming, 4 (1973), pp. 193-201.

[11] J. S. PANG, On the convergence of dual ascent methods for large-scale linearly constrained optimization problems, Univ. of Texas at Dallas, unpublished manuscript, 1984.

[12] R. W. COTTLE AND J. S. PANG, On the convergence of a block successive over-relaxation method for a class of linear complementarity problems, Math. Programming Stud., 17 (1982), pp. 126-138.

[13] D. P. BERTSEKAS AND S. K. MITTER, A descent numerical method for optimization problems with nondifferentiable cost functionals, this Journal, 11 (1973), pp. 637-652.

[14] L. R. Ford, JR. AND D. R. Fulkerson, Flows in Networks, Princeton Univ. Press, Princeton, NJ, 1962.

[15] C. H. Papadimitriou and K. Steiglitz, Combinatorial Optimization: Algorithms and Complexity, Prentice-Hall, Englewood Clifts, NJ, 1982.

[16] J. EDMONDS AND R. KARP, Theoretical improvements in algorithmic efficiency for network flow problems, J. Assoc. Comput. Mach., 19 (1972), pp. 248-264.

[17] P. TSENG, Relaxation methods for monotropic programming problems, Ph.D. thesis, Operations Research Center, Massachusetts Inst. of Technology, 1986.

[18] D. KLINGMAN, A. NAPIER AND J. STUTZ, NETGEN-A program for generation large scale (un)capacitated assignment, transportation and minimum cost network problems, Management Sci., 20 (1974), pp. 814-822.

[19] S. ZENIOS AND J. M. MULVEY, Simulating a distributed synchronous relaxation method for convex network problems, Working Paper, Department of Civil Engineering, Princeton Univ., January 1985.

[20] D. P. Bertsekas, Distributed relaxation methods for linear network flow problems, Proc. of 25th Conf. on Decision and Control, Athens, Greece, December 1986.

[21] _ Distributed asynchronous relaxation methods for linear network flow problems, LIDS Report P-1606, Massachusetts Inst. of Technology, Cambridge, MA, September 1986. 